\title{
Strategies for Choosing Descent Flight-Path Angles for Small Jets
}

\author{
Minghong G. Wu* \\ University of California, Santa Cruz, Moffett Field, California, 94035-1000 \\ Steven M. Green ${ }^{\dagger}$ \\ NASA Ames Research Center, Moffett Field, California, 94035-1000
}

\begin{abstract}
Three candidate strategies for choosing the descent flight path angle (FPA) for small jets are proposed, analyzed, and compared for fuel efficiency under arrival metering conditions. The strategies vary in operational complexity from a universally fixed FPA, or FPA function that varies with descent speed for improved fuel efficiency, to the minimum-fuel FPA computed for each flight based on winds, route, and speed profile. Methodologies for selecting the parameter for the first two strategies are described. The differences in fuel burn are analyzed over a year's worth of arrival traffic and atmospheric conditions recorded for the Dallas/Fort Worth (DFW) Airport during 2011. The results show that the universally fixed FPA strategy (same FPA for all flights, all year) burns on average 26 lbs more fuel per flight as compared to the minimum-fuel solution. This FPA is adapted to the arrival gate (direction of entry to the terminal) and various timespans (season, month and day) to improve fuel efficiency. Compared to a typical FPA of approximately $3^{\circ}$, the adapted FPAs vary significantly, up to $1.3^{\circ}$ from one arrival gate to another or up to $1.4^{\circ}$ from one day to another. Adapting the universally fixed FPA strategy to the arrival gate or to each day reduces the extra fuel burn relative to the minimum-fuel solution by $27 \%$ and $34 \%$, respectively. The adaptations to gate and time combined shows up to $57 \%$ reduction of the extra fuel burn. The second strategy, an FPA function, contributes a $17 \%$ reduction in the 26 lbs of extra fuel burn over the universally fixed FPA strategy. Compared to the corresponding adaptations of the universally fixed FPA, adaptations of the FPA function reduce the extra fuel burn anywhere from 15-23\% depending on the extent of adaptation. The combined effect of the FPA function strategy with both directional and temporal adaptation recovers $67 \%$ of the extra fuel relative to the minimum-fuel solution.
\end{abstract}

\section{Nomenclature}

$\gamma \quad$ Inertial flight-path angle

$\gamma_{0} \quad$ Parameter of Strategy 2

$\gamma_{\text {univ }} \quad$ Parameter of Strategy 1

CAS Calibrated airspeed

CDA Continuous Descent Arrival

DCAS Descent calibrated airspeed

EDA Efficient Descent Advisor

FMS Flight Management Systems

FAA Federal Aviation Administration

FPA (Inertial) flight-path angle

NAS National Airspace System

RUC Rapid Update Cycle

SBANY Speed-Brake-Any

SB0 Speed-Brake-Zero

\footnotetext{
*Research Engineer, AIAA Member
}

${ }^{\dagger}$ Aerospace Engineer, AIAA Member 
TMA Traffic Management Advisor

TOD Top of descent

TS Trajectory Synthesizer

VNAV Vertical Navigation

\section{Introduction}

Trajectory management of metered arrivals into high-density terminal airspace is a critical component for NextGen Trajectory Based Operations concepts. ${ }^{1,2}$ Significant research has focused on the utilization of modern flight management systems (FMS) to enable continuous descent planning, at least from cruise to a metering fix within the the Terminal Radar Approach Control Facilities (TRACON) airspace in the United States or the Terminal Control airspace in other countries. Field trials over the past two decades have provided a great deal of insight into the ability to predict and execute such continuous descents for major-carrier type aircraft such as Boeing and Airbus. ${ }^{3-5}$ However, little attention has been paid to "small" (regional, business and light) jet types, which comprise a large and potentially high-growth portion of NextGen traffic operations. ${ }^{6}$

Unlike the larger aircraft types, which are equipped with performance-based FMS systems that attempt to optimize the vertical profile with near-idle descents, the smaller jets are equipped with simpler Vertical Navigation (VNAV) capabilities. Descent planning for these types typically involves a fixed-flight path angle (FPA) descent that is either based on a company-programmed default or a pilot-selected value. For example, the "standard operating procedure" of one large regional carrier called for an indicated airspeed of 320 knots for descent, initiated at the cruise Mach number, using a default FPA of $-3.8^{\circ}$. This works fine for nominal conditions with light to moderate winds and no Air Traffic Control interruptions to the descent. However, when speed restrictions are issued by controllers for metering and spacing, the nominal descent plan can become inefficient and difficult, if not sometimes impossible, to fly in strong tailwinds. In addition, random observations of regional jet operations and pilot interviews revealed that a large variety of descent-planning techniques are used by pilots, even for the same equipment. These techniques vary in terms of the selection of descent angle, bottom-of-descent planning, and top-of-descent transition. Sometimes they take into account winds aloft and weight, but rarely descent speed.

It is important to develop and standardize the procedures for establishing efficient descent FPAs for small jets. Such standardized procedures lead to better trajectory predictability and provide benefits for separation assurance. ${ }^{7}$ However, selecting the FPA is a non-trivial task, as the most fuel-efficient and "flyable" FPAs ${ }^{\mathrm{a}}$ can vary significantly as a function of aircraft type, weight, speed profile, and, particularly, winds and wind gradient. ${ }^{8}$ The systematic effect of these variables on the selection of fuel-efficient and flyable FPAs is far from understood, and only a limited analysis of fixed-FPA descents exists in the literature. ${ }^{9-12}$ Under some conditions, a steep descent may be the most fuel efficient and yet be operationally unacceptable to pilots. Even if a steep descent is achievable with the utilization of speed brakes, many pilots are reluctant, if not unwilling, to use them because of noise and ride discomfort. Given the significant variation in the winds aloft from one area of the National Airspace System (NAS) of the United States to another, and from one day, week or month to another, the FPA procedure may need to be "adaptive".

The purpose of this paper is to propose several candidate methodologies for selecting descent FPAs for small jets, and analyze their impact on the FPA and fuel efficiency. In this work, three candidate strategies are applied to a full year's worth of arrival traffic and recorded winds data for Dallas/Fort Worth (DFW) Airport from the year 2011. DFW was selected because of its large seasonal variation in winds aloft, the eastwest symmetry of it's arrival routes which accentuates head/tail-wind differences, and the density of regional jet operations. The benefits of various adaptations of these strategies are investigated as a function of the arrival gate and periods of time (categorized by season, month and day). The rest of the paper is organized as follows: Section II describes the metered environment; Section III outlines the three proposed FPA strategies and the methodology for parameter selection; Section IV describes the adaptation of Strategies 1 and 2 as a function of location (arrival gate) and time; Section V describes the approach taken to model the descent conditions and the metering-constrained arrival trajectories; Section VI presents results of the selected FPAs in various adaptations and compares fuel burn among the three strategies; Section VII investigates the effect

\footnotetext{
${ }^{a}$ In this paper, an FPA is considered flyable if the aircraft can maintain both the FPA and the descent speed necessary to meet its assigned metering time.
} 
of wind on the results, focusing on data from a few selected days for further insight; and, finally, Section VIII summarizes the findings.

\section{Background}

In the United States, the Traffic Management Advisor (TMA) ${ }^{13,14}$ computes metering-fix scheduled times of arrival (STA) at the TRACON boundary in order to control throughput of en-route traffic arriving at a high-density airport. The Efficient Descent Advisor (EDA), which was developed to assist en-route controllers in achieving TMA's STAs while maintaining separation, computes clearance advisories that also enable fuel-efficient continuous descent arrivals (CDA). ${ }^{15-20}$

Consider an arrival that is guided by a controller using EDA to plan and execute a continuous descent in order to cross a TRACON metering fix at the STA specified by TMA. During periods of congestion, this STA will result in a small delay at the metering fix to keep the TRACON arrival traffic manageable. ${ }^{14}$ Depending on the conditions for each aircraft, speed reductions are typically able to absorb three to four minutes of delay for flights about 20 minutes or $150 \mathrm{nmi}$ from the metering fix. ${ }^{18}$ While other maneuvers such as temporary altitude clearances or path stretches are required for larger delays, it is the speed advisories that are relevant to the analysis presented below.

The previous development and testing of EDA focused primarily on descent procedures for large jets equipped with a performance-based FMS. The last field trial conducted at Denver Center in the fall of 2010 (results yet to be published) began to explore the issues related to small jets. While a simple, fixed-FPA descent procedure using prescribed clearances was introduced for the purposes of that field trial, EDA itself still lacks a defined descent procedure and corresponding algorithm for defining the descent FPA for small jets. The strategies presented here for selecting descent FPAs will complement the current EDA capabilities and support fuel-efficient and predictable arrival-metering operations for small jets.

\section{Three FPA Selection Strategies}

In a previous paper, three strategies were proposed to define the fixed FPA for small jet arrivals under metering conditions. ${ }^{8}$ Monte Carlo simulations using simplified wind distributions were performed to evaluate these strategies. The three strategies were as follows:

1. Universally fixed FPA

2. FPA as a function of descent speed

3. Minimum-fuel FPA for every flight (Referred to as "Custom FPA" in the previous paper)

Each strategy is discussed in more details in the following sections.

\section{III.A. Strategy 1: Universally Fixed FPA}

Strategy 1 defines a universal FPA for all small jets arriving to an airport, gate, or route. This is akin to a glide slope extended from the arrival metering fix back up to the top of descent (TOD) in the en-route airspace. The idea of a universally fixed-FPA descent procedure was explored by Tong et al. at Boeing, with a focus on modifications required for the performance-based FMS equipped jets. ${ }^{9}$ The difference between Strategy 1 and the work of Tong et al. is twofold. First, Strategy 1 applies to small jets whose VNAV can guide fixed-FPA descents, while the work of Tong et al. attempts to enable a fixed FPA procedure for jets equipped with a performance-based FMS. Second, methods are developed in this paper to adapt the universally fixed FPA in Strategy 1 to season, month, day, or arrival gate.

The advantage of Strategy 1 is its simple form, which allows it potentially to be published as part of the arrival procedure. The disadvantage is that it does not account for the effects of descent speed and winds on the fuel efficiency or the range of flyable FPAs.

\section{III.B. Strategy 2: FPA as a Function of the Descent Speed}

Strategy 2 takes into account the effects of the descent speed on the range of fuel-efficient and flyable FPAs. Table 1 summarizes an example of the FPA as a function of the descent calibrated airspeed (CAS), 
designed for an EDA flight trial held in Denver Center in 2010 for regional jets operated by Skywest Airlines. Note that the descent CAS in Table 1 ranges from 250 knots to 320 knots. The low end is the minimum speed

Table 1. The FPA function used in the flight trial at Denver Center in 2010

\begin{tabular}{cc}
\hline Range of Descent CAS (knots) & $\mathrm{FPA}\left(^{\circ}\right)$ \\
\hline $250-260$ & -2.8 \\
$270-280$ & -3.1 \\
$290-300$ & -3.4 \\
$310-320$ & -3.8 \\
\hline
\end{tabular}

controllers are allowed to issue without consulting the pilot. The high end represents the Skywest Airlines' preferred descent speed for their Bombardier CRJ aircraft. Participating Skywest pilots determined the FPA to fly by using the descent CAS issued by the EDA clearance. Table 1 was developed in collaboration with Skywest, and validated in a piloted simulation at their training facility. The specific values were selected with consideration of flyability, avoiding the use of speed brake or relatively high power settings, and fuel efficiency. Although it was adequate for the purpose of the flight trial, the table's sparse values of FPA leave large gaps in the achievable ranges of time-to-fly. Also, the FPA function in Table 1 was static and not adapted for winds aloft and the direction of flight.

In our previous work, a finer-grained, step-wise function with an adaptive parameter was used. ${ }^{8}$ In contrast to the FPA function used in the field trial, this FPA function changes the FPA by $0.1^{\circ}$ for every 10 knots of descent CAS. It was observed that this rate of change of FPA with respect to the descent CAS reflects the variation of fuel-efficient FPAs with speed. ${ }^{8}$ Moreover, the small increment of $0.1^{\circ}$ leads to smaller gaps in the achievable ranges of time-to-fly. The FPA function is defined by Equation 1:

$$
\gamma= \begin{cases}\gamma_{0} & , \text { if DCAS }<255 \\ \gamma_{0}-0.1 & , \text { if } 255 \leq \text { DCAS }<265 \\ \gamma_{0}-0.2 & , \text { if } 265 \leq \text { DCAS }<275 \\ \ldots, & \end{cases}
$$

where $\gamma$ is the FPA in degrees, DCAS is the descent CAS in knots, and the adaptive parameter $\gamma_{0}$ stands for the value of $\gamma$ at 250 knots of the descent CAS. Note that the increment of $0.1^{\circ}$ matches the precision of FPA-selection increments typical of small jet avionics. Also note the selection of the parameter $\gamma_{0}$ defines the entire FPA function. To select an FPA for an arrival flight, the range of time (i.e., time-to-fly) achievable by the speeds mapping to each FPA is computed, and the FPA whose resulting range of time covers the STA is selected.

One advantage of Strategy 2, like Strategy 1, is that the function can be determined ahead of time and therefore published as part of the arrival procedure. Moreover, by taking into account the descent speed, Strategy 2 is expected to be more fuel-efficient than Strategy 1. However, like Strategy 1, Strategy 2 has the disadvantage that it does not account for the effects of the direction of the winds aloft on the range of fuel-efficient and flyable FPAs.

\section{III.C. Strategy 3: Minimum-Fuel FPA}

While Strategies 1 and 2 define the FPA and the FPA function ahead of time, Strategy 3 selects a computed minimum-fuel FPA to be communicated explicitly to the pilot of each flight just prior to the TOD. The computation is based on the route, winds and temperature aloft, and the speed profile necessary to meet the STA. Figure 1 sketches the fuel burn as a function of the descent FPA for a typical flight.

Strategy 3, by definition, selects the minimum-fuel FPA for each arrival flight and therefore is better than Strategies 1 and 2 in terms of fuel saving. It serves as a reference of the fuel burn to compare with the first two strategies. However, the requirement of communicating the FPA to the pilot in real time may make it too complex to implement in the near term. 


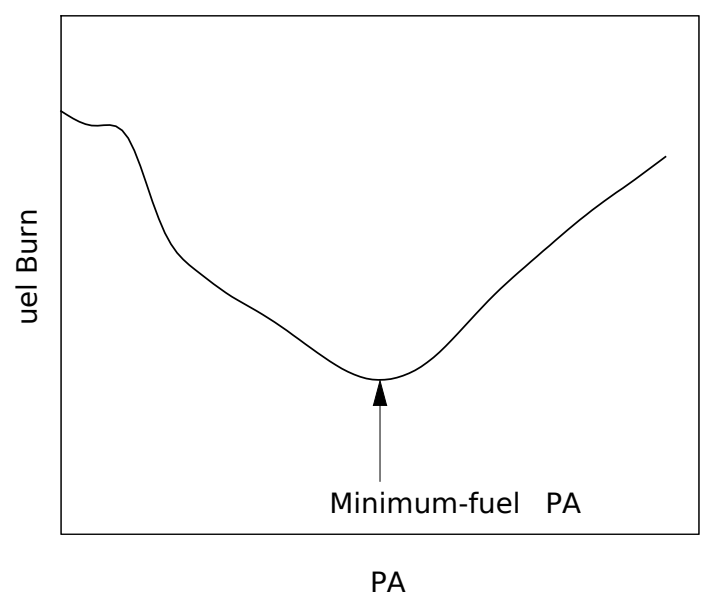

Figure 1. Strategy 3 selects the minimum-fuel FPA.

\section{III.D. Planned Speed-Brake Usage}

In addition to predicted fuel burn, the selection of FPA should also take into account uncertainties in vertical profile planning. To keep the aircraft on the planned vertical path, power adjustment is preferred to speed-brake deployment for reasons of passenger comfort and the desire of pilots to reserve the use of a speed brake for rare occasions. Besides, some aircraft types have less effective speed brakes than others. Two levels of planned-speed-brake usage are modeled in our analysis of feasible meet-time trajectories to explore the impact of speed-brake usage on the selection of descent FPA and resulting fuel burn. In one condition, FPAs are limited to those that do not require speed-brake usage. In the other, any FPA is considered valid as long as the estimated amount of speed-brake usage is within the modeled speed brake capacity for that aircraft. These conditions are imposed on the acceptable predicted trajectories in the analysis. Actual speed-brake usage required in flight might vary based on the difference between the actual and the predicted state of the flight and atmosphere.

\section{III.E. The Parameters in Strategies 1 and 2}

The universally fixed FPA in Strategy 1 and the $\gamma_{0}$ in Strategy 2 are parameters that must be selected carefully, with consideration for the prevailing winds aloft and, to a lesser extent, the anticipated traffic demand. Our approach is to use fast-time simulation to analyze the fuel efficiency and flyability of the FPAs resulting from the parameters selected for each strategy. On the one hand, the resulting FPA should not be so shallow as to waste fuel unnecessarily. On the other hand, the FPA should not be so steep as to require excessive use of speed brakes to maintain both the assigned descent speed and FPA throughout the descent. For the purposes of this work, it is assumed that reasonably accurate estimates of the prevailing winds and arrival traffic demand are available. This assumption will be further discussed in Section IV.

The following criteria have been applied in previous work ${ }^{8}$ to select the universally fixed FPA, denoted as $\gamma_{\text {univ }}$, in Strategy 1, and $\gamma_{0}$ in Strategy 2:

1. The parameter must be selected such that at least $99 \%$ of the flights do not exceed the limit for the planned speed-brake usage.

2. The parameter must be selected such that, without violating the previous criterion, it results in the least average fuel burn per flight.

The first criterion defines the steepest parameter that can be selected, and the second criterion selects a minimum-fuel FPA from those parameters no steeper than the steepest parameter allowed by the first criterion. These same criteria are used for the analysis in this work. 


\section{Adaptation of Strategies 1 and 2}

\section{IV.A. Adaptation to Gates and Timespans}

A practical implementation of Strategies 1 or 2 would be to adapt their parameters, $\gamma_{\text {univ }}$ and $\gamma_{0}$, to specific airports, in order to account for the prevailing winds and local traffic flows. As discussed in Section III.E, the selection of the parameter(s) should be such that the resulting descent FPAs are flyable across significant variations of wind along the route. For airports with opposing arrival directions, particularly the classical four-corner-post configuration, strong winds become problematic. While steeper FPAs are typically more fuel-efficient for flights in a headwind, they may be unflyable for flights in the opposite direction. To ensure that $\gamma_{\text {univ }}$ or $\gamma_{0}$ leads to flyable descents over a wide range of arrival routes and wind conditions, the static implementation must be conservative in its selection. This implies selecting a shallow, relatively fuel-inefficient $\gamma_{\text {univ }}$ or $\gamma_{0}$.

These observations motivated the adaptation of $\gamma_{\text {univ }}$ and $\gamma_{0}$ to the direction of flight. Such an adaptation would define a distinct $\gamma_{\text {univ }}$ or $\gamma_{0}$ for each arrival gate feeding an adapted airport. Compared to the static implementation, adaptation to the direction of flight provides a greater degree of "customization" and the potential to improve fuel efficiency.

While adaptation to the arrival direction reduces the variation of along-track winds due to directions, it does not mitigate the variation of winds over time. In many areas of the United States that are in the path of the jet stream, seasonal variation of winds aloft can be significant in both magnitude and direction. Therefore, the fuel efficiency of Strategies 1 and 2 can be further improved by adapting $\gamma_{\text {univ }}$ and $\gamma_{0}$ to each season, month, or even each day. A good selection of $\gamma_{\text {univ }}$ and $\gamma_{0}$ for a period of time relies primarily on the quality of the estimated (or forecast) winds aloft and, to a lesser extent, the estimated traffic demand. While quality forecasts of winds aloft beyond 24 hours may be problematic, estimates of the winds aloft over a period of time, such as a season or a month, may be possible through climatological and historical analysis. The approach here is to assume the availability of reliable estimates or forecasts of winds aloft for the selection of the FPA or FPA function for various adaptations. Although the actual fuel benefits for adaptations to longer time horizons will be less than that estimated by this analysis, the trends are insightful.

\section{IV.B. Dallas/Fort Worth Airport}

A full year's worth of Fort Worth Air Route Traffic Control Center's arrival traffic during 2011 was selected for our analysis. Figure 2 shows tracks of arrival flights of small jets on July 28th, 2011. Each arrival flight enters the TRACON through one of the NE, SE, SW, and NW gates. All flights of the small jets were assumed to arrive at the Dallas/Fort Worth (DFW) airport. ${ }^{\mathrm{b}}$ The primary metering fixes for these four gates are KARLA (NE), HOWDY (SE), DEBBB (NW), and FEVER (SW). Although there are other metering fixes for each gate, it is assumed in this analysis that the arrival flights all go through the primary metering fixes. Therefore, the names KARLA, HOWDY, DEBBB, and FEVER will be used interchangeably with the names of the gates.

The Rapid Update Cycle (RUC) weather forecast ${ }^{21}$ serves as an estimate of the "true" winds and temperatures aloft. Figure 3 illustrates a typical variation of wind across the four arrival gates. This figure presents the probability distribution of tailwinds during the year of 2011 along a straight path to each gate starting from a central location, $150 \mathrm{nmi}$ upstream of the metering fix, at 35,000 ft. The four paths selected for measuring the probability are shown as red lines in Figure 2. In general, the winds in winter are stronger. Winds experienced in the eastern gates have similar distributions; winds experienced in the western gates also have similar distributions. Between January and March, winds for KARLA and FEVER show slightly wider distribution than the winds for HOWDY and DEBBB, respectively. Note that the distribution of winds for KARLA in the JAN-MAR timespan shows a small probability of a tailwind. Similarly, a small probability of a headwind for FEVER is observed. Independent inspection of some of the RUC data files revealed a predominantly west wind that prevailed in the first three and the last two months of 2011 . These conditions result in strong headwinds for flights coming through the NE and SE gates during these periods of time, and strong tailwinds for the western gates. The strong west winds during the winter months are typical of the subtropical jet stream at high altitudes. The jet stream moves north in the summer, and so the wind magnitude reduces in July, August, and September, to 50 knots or less most of the time for all gates.

\footnotetext{
${ }^{\mathrm{b}}$ The contribution of the arrival small jets to the other airports in the TRACON is negligible.
} 


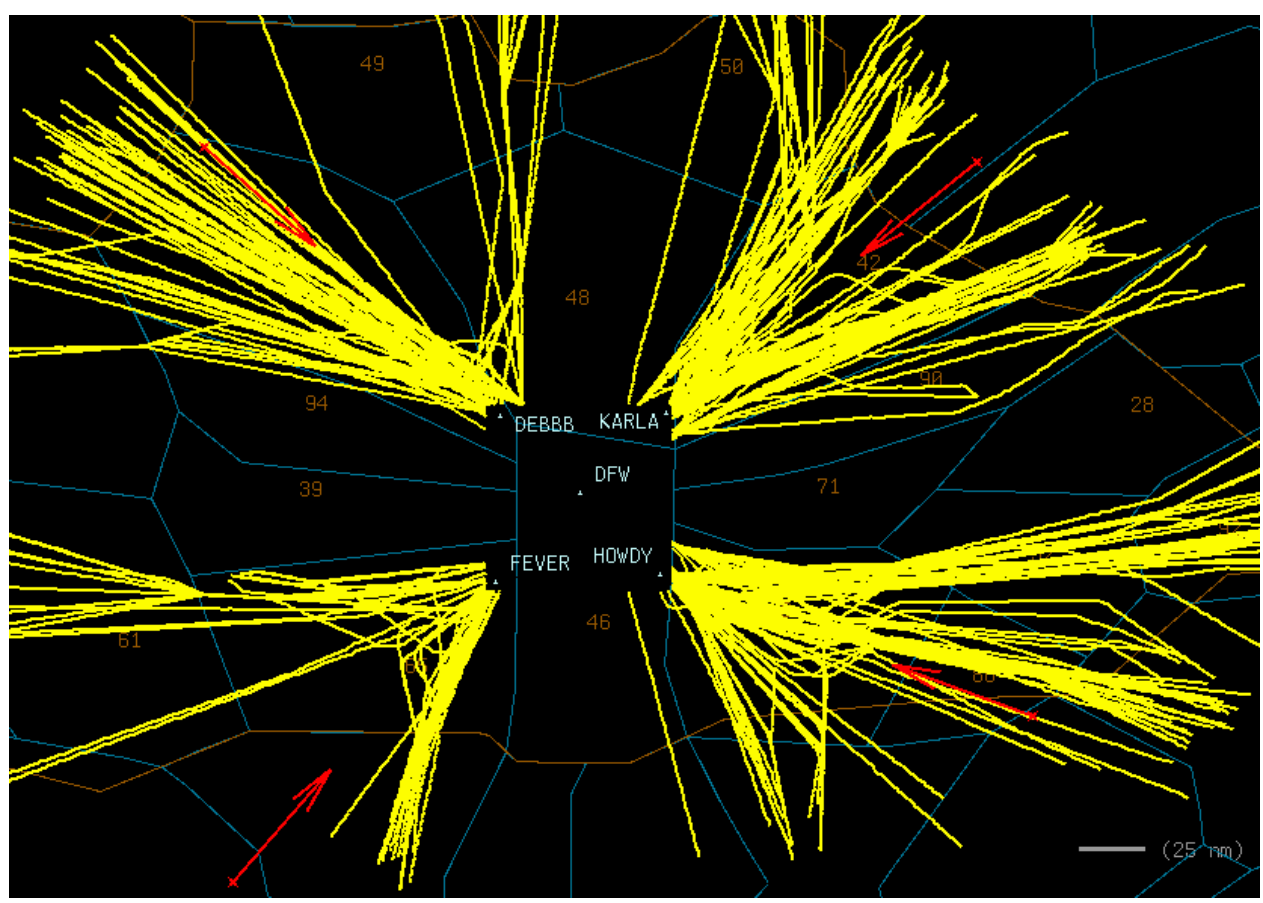

Figure 2. Tracks of arrival small jets into DFW on July 28th, 2011.

\section{IV.C. Adaptation Types}

We propose a set of adaptations, organized as in Table 2. The columns represent different levels of

Table 2. The types of adaptations categorized by their granularity in location and time

\begin{tabular}{|c|c|c|c|c|}
\hline & \multicolumn{5}{|c|}{ Airspace/Direction } \\
\hline Time & NAS & Airport & Arrival Gate & Arrival Route \\
\hline Static & & 1 & 5 & \\
\hline Season & & 2 & 6 & \\
\hline Month & & 3 & 7 & \\
\hline Day & & 4 & 8 & \\
\hline Hour & & & & \\
\hline
\end{tabular}

adaptation for different airspaces, starting with a basic "one size fits all" adaptation for all airports across the NAS. Moving to the right, each column represents a progressively finer adaptation of the FPA strategy to a specific airport, individual arrival gates (corner posts) feeding an airport, all the way down to specific arrival routes feeding each arrival gate. The rows represent a temporal scale starting at the top with the simplest option of a static adaptation. Moving down, each row represents a progressively finer adaptation to account for changes in the prevailing winds as a function of season, month, day or even hour. The table illustrates the overall approach and potential scope. For the purposes of this paper, the analysis will assess the eight types highlighted and numbered in the table. The analysis of adaptations at the NAS-wide level, or at the level of specific arrival routes and/or hours of the day, are left for future work. Table 3 lists the names of these eight adaptation types analyzed in this work:

Section $\mathrm{V}$ describes the methodologies for the analysis, modeling details, and methods for selecting the FPA and FPA function for each adaptation. 

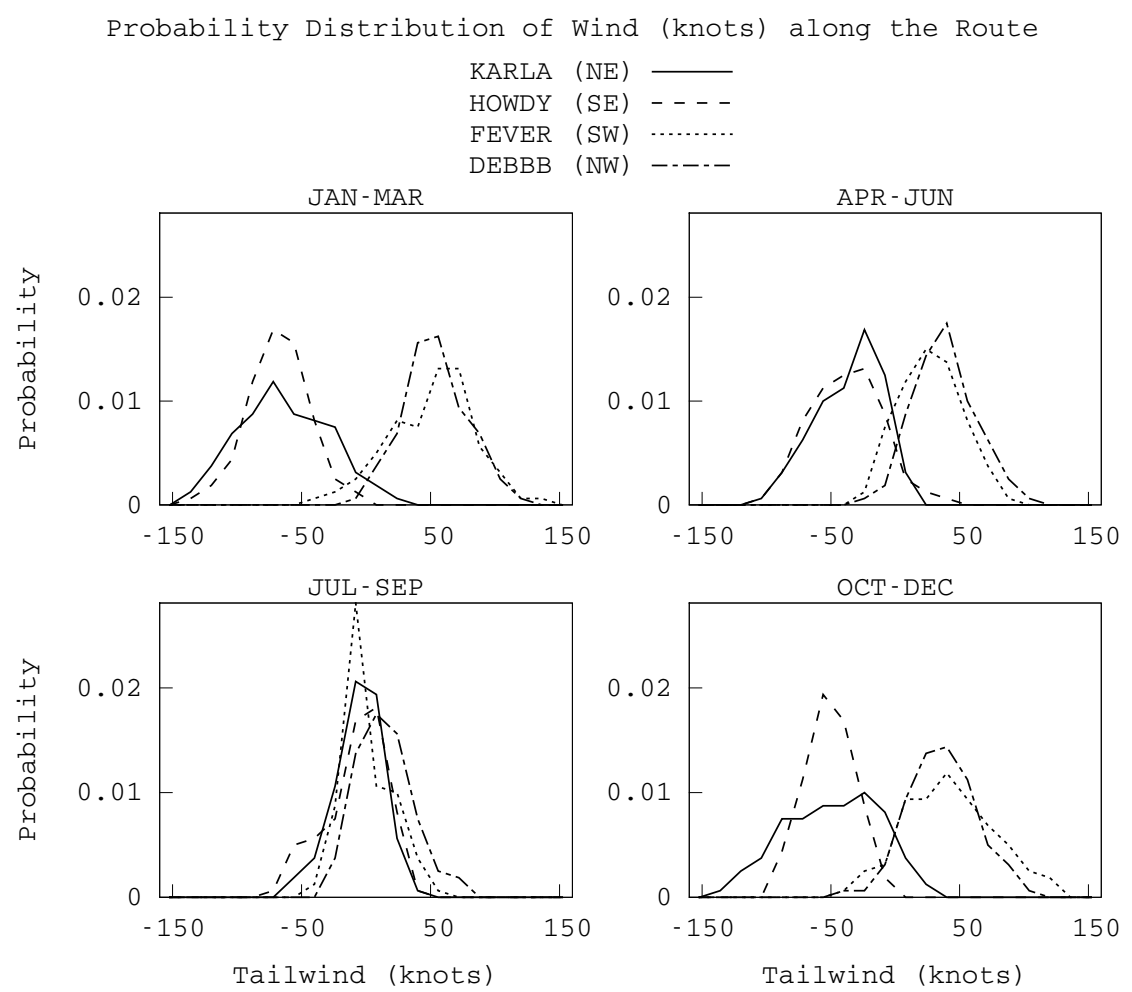

Figure 3. Wind along typical arrival routes at $35,000 \mathrm{ft}$ in Fort Worth Center, 2011. Note that a negative tailwind represents a headwind.

Table 3. The eight adaptation types analyzed for the DFW traffic

\begin{tabular}{lll}
\hline$\#$ & Name & FPA/FPA Function Defined for \\
\hline 1 & Airport-Static & the airport \\
2 & Airport-Season & the airport by season \\
3 & Airport-Month & the airport by month \\
4 & Airport-Day & the airport for each day \\
5 & Gate-Static & each arrival gate \\
6 & Gate-Season & each arrival gate by season \\
7 & Gate-Month & each arrival gate by month \\
8 & Gate-Day & each arrival gate for each day \\
\hline
\end{tabular}

\section{Analysis Approach}

\section{V.A. Calibrating and Comparing the Three Strategies}

A methodology was developed in the previous work ${ }^{8}$ to compare the benefits of the three strategies of selecting the descent FPA. Conceptually, this methodology consists of two parts: first select the parameters for Strategies 1 and 2, and then compare the fuel burn between the three strategies for a set of traffic and wind conditions.

The first part of the methodology determines the parameters of Strategies 1 and 2 using estimates of winds and temperatures aloft, traffic demand, and modeled metering delays:

- For each flight and each candidate value of the parameter, compute the (predicted) fuel burn and planned speed-brake usage.

- Select the value of the parameter based on the results, using the criteria defined in Section III.E for 
speed-brake usage and fuel burn.

- Repeat the above steps for both Strategies 1 and 2.

The second part of the methodology estimates and compares the fuel burn of the strategies, using the estimates of winds and temperatures aloft, actual traffic data, and modeled or actual metering delays:

- Compute the fuel burn and planned speed-brake usage for each flight using Strategies 1 and 2, respectively.

- Compute the fuel burn and planned speed-brake usage for each flight using Strategy 3.

- Compare the fuel burn of Strategies 1 and 2 relative to Strategy 3.

- Iterate over all flights, compute the average fuel burn penalty of Strategies 1 and 2.

While the estimates of winds, temperatures aloft, and traffic demand for the second part need not be the same as that used for the first step, they are the same in this analysis, being the RUC data and the track data of DFW recorded in 2011. This means the parameters of Strategies 1 and 2 are selected as best as they can be, using the same wind and track information accessible in real time to Strategy 3. The fuel benefits of Strategies 1 and 2 obtained in this way can be viewed as an upper bound of the actual relative benefits. Since the same estimates of winds and temperatures aloft and traffic demand were used in both parts of the methodology, the fuel burn and planned speed-brake usage recorded for the first part can be directly used in step 1 of the second part without having to recalculate them.

The implementation of the methodology further simplifies the data collection process by combining its two conceptual parts into one run of a fast-time simulation as described in detail in the following sections. The basic idea is that, for each arrival flight and a modeled metering delay, the analysis computes a set of meet-time trajectories with varying FPA and descent CAS profile. Because the FPA selected by each strategy must be from these trajectories, the analysis of these trajectories was sufficient for the fuel burn comparison among the three strategies.

The following sections describe modeling of the trajectories, metering delays, meet-time trajectories, parameter selection for the adaptations of Strategies 1 and 2, and fuel burn comparison.

\section{V.B. Route and Vertical Profile}

A distance-based freeze horizon of $160 \mathrm{nmi}$ was assumed, inside of which TMA would fix the STA for the aircraft. ${ }^{13}$ The initial condition was selected at a corresponding track point. For flights following shorter arrival routes from the north and the south, the first track point in the Center was selected. For simplification, Direct-To trajectories from the initial point to the primary metering fix of a gate were assumed without actually parsing the flight plans for the waypoints. The initial altitude must be at least 16,000 ft for the flight to be considered for the analysis. Metering fix crossing conditions of 11,000 ft and 250 knots in CAS were assumed.

Figure 4 illustrates a typical vertical profile that has five segment types. Individual trajectories will contain all or a subset of these segments depending on the speed profile needed to meet the STA. Each segment is modeled by fixing two control parameters. One of the parameters is the FPA; the second depends on the segment. For a cruise segment the model fixes the airspeed or the engine control for acceleration or deceleration. For the constant speed descent segments, the model fixes an airspeed in Mach or CAS.

\section{V.C. Aircraft Modeling}

The Trajectory Synthesizer (TS) component ${ }^{22,23}$ of the Center-TRACON Automation System (CTAS) ${ }^{24}$ was used to compute the trajectories and their associated fuel burn and planned speed-brake usage. While a detailed performance model of small jet types would have been desirable, one was not available. Instead, a high-fidelity, CTAS model for a mid-size, narrow-body, twin-jet airliner with a typical descent weight of 170,000 lbs was used. The speed envelopes were selected within the ranges of small jets. An empirical constant was used to model the maximum drag coefficient resulting from speed-brake deployment. ${ }^{8}$ Weight uncertainty was modeled by a normal distribution with a deviation of $8,400 \mathrm{lbs}$. 


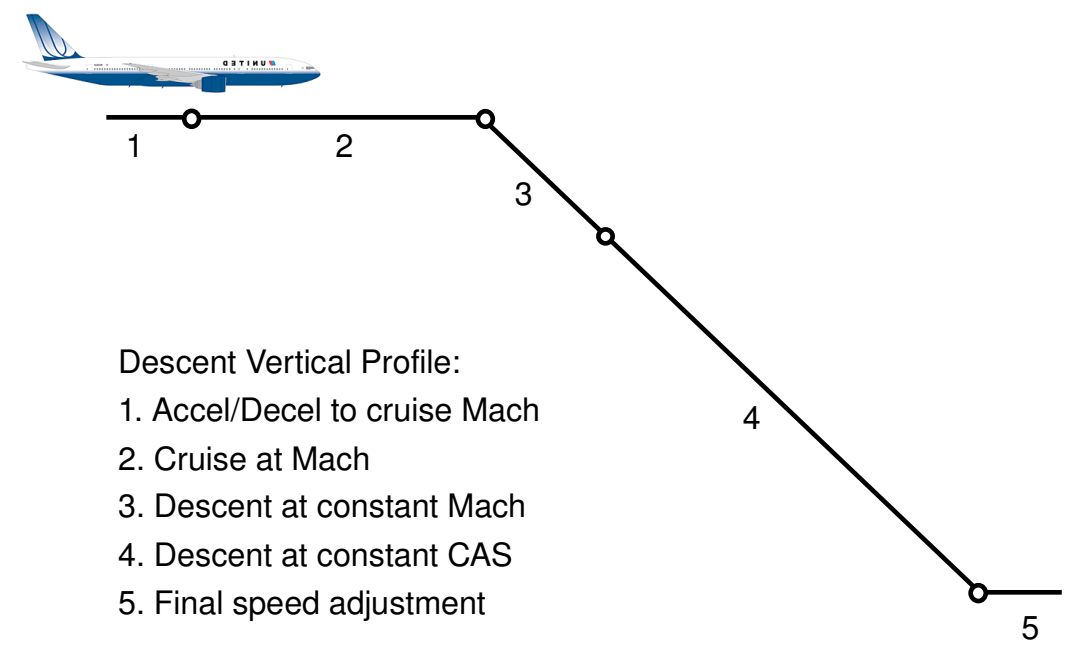

Figure 4. A general vertical profile that contains five segment types.

To account for the variation of the fuel-burn rate among aircraft types, the fuel burn was scaled by the empirical formula

$$
f_{i}=f_{0} * \frac{N_{i}+30}{230}
$$

where $f_{i}$ is the scaled fuel burn; $f_{0}$ is the raw fuel-burn rate calculated by CTAS for the mid-size, narrowbody, twin-engine jet; and $N_{i}$ is the number of passengers' seats typical of the aircraft type $i$. This empirical formula was derived by taking the linear regression of the nominal cruise fuel-burn rate of eight small jets plus the mid-sized twin engine jet, using the Base of Aircraft Database (BADA) 3.8 performance model. ${ }^{25}$ Although BADA provides modeling parameters for small jets, the calibration of these parameters focused on nominal flight conditions only. Since the analysis in this work explored a wide range of the speeds, containing both nominal and off-nominal ones, it was decided that the high-fidelity CTAS model with scaled fuel burn was more appropriate for the fuel-burn analysis.

\section{V.D. Metering Delay}

The delay at the metering fix was modeled by a uniform distribution between zero and the maximum delay that can be absorbed by speed reductions. The delay time was added to the nominal time in order to specify the STA. In the absence of a "standard" FPA for defining the nominal trajectory, the idle-thrust descent was used for defining the nominal and slow-limit trajectories. The descent CAS of 320 knots was assumed as the airline-preferred descent CAS and was used, along with an idle thrust descent and the aircraft's initial cruise speed in defining the nominal time-to-fly. To define the slowest time-to-fly, the minimum cruise and descent speeds of along with the idle thrust descent were used. The maximum delay is the difference between the slowest time-to-fly and the nominal time-to-fly.

\section{V.E. Fixed-FPA Meet-Time Trajectories}

A set of meet-time trajectories with varying FPA and descent CAS profile was computed for each flight using a modeled metering delay. All the meet-time trajectories must satisfy the allowed planned speed-brake usage. Fuel burn and planned speed-brake usage was calculated for each meet-time trajectory. It turned out that these meet-time trajectories provided all the fuel-burn data needed for comparison of the three strategies, because each of the three strategies must select an FPA from these meet time trajectories.

For descent FPAs ranging from $-1.8^{\circ}$ to $-5.5^{\circ}$, with an increment of $0.1^{\circ}$, a meet-time algorithm attempted to compute a fixed-FPA trajectory for each value of the FPA. The meet-time algorithm iterated cruise and descent speeds until the trajectory met the desired time-to-fly within a tolerance of 2 seconds. Cruise and descent speeds were related by the Cruise-Equals-Descent speed mode developed for EDA, ${ }^{18}$ designed based on operational considerations. Note that this speed mode does not guarantee the best fuel efficiency in the trajectories. ${ }^{26}$ 
Figure 5 shows a typical range of FPA-descent-speed combinations defining the set of meet-time trajectories for a flight meeting an STA, labeled as "STA1". Each symbol represents the pair of descent CAS and FPA of a meet-time trajectory. The steepest FPA in this set of meet-time trajectories is $-3.6^{\circ}$. Trajectories steeper than $-3.6^{\circ}$ were not included, because their predicted speed-brake usage exceeded the allowed planned speed brake usage.

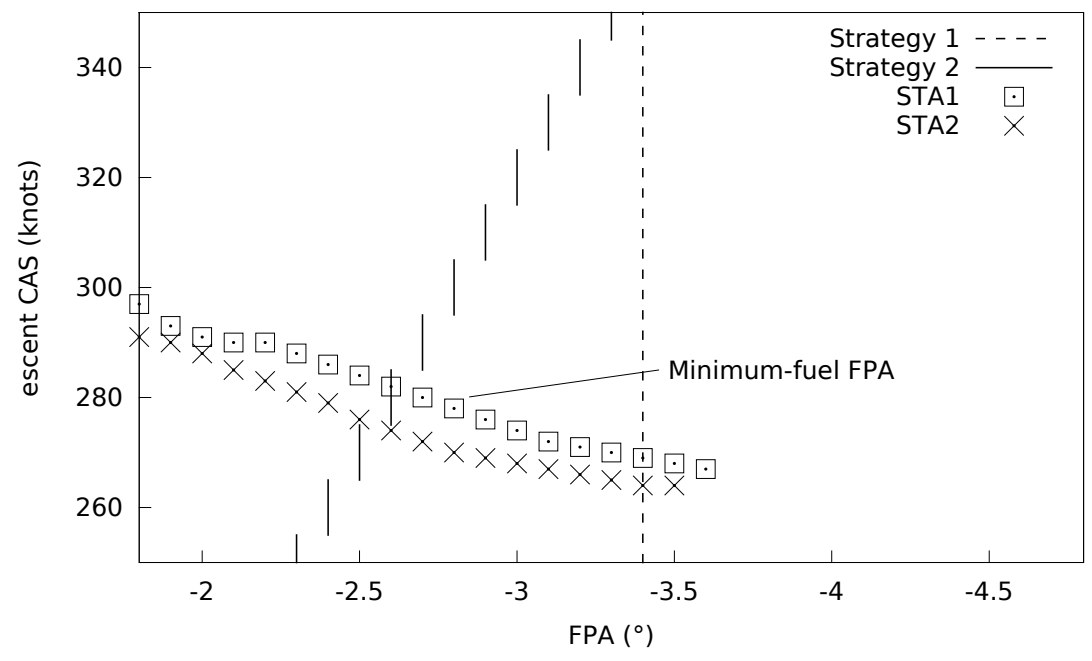

Figure 5. Applying Strategies 1 and 2 to the selection of FPAs.

To illustrate how the descent FPA may be selected differently by the three strategies for the "STA1" flight, suppose Strategy 3 is used for selecting the FPA. Strategy 3 selects the FPA of the minimum-fuel trajectory, which is found to be $-2.8^{\circ}$. Now suppose Strategy 1 with an $\gamma_{\text {univ }}$ of $-3.4^{\circ}$ is used. A schematic representation of Strategy 1 is shown as the dashed vertical line in Figure 5. The resulting meet-time trajectory with the selected $\gamma_{\text {univ }}$ of -3.4 must belong to the set of meet-time trajectories. Therefore, the fuel burn as a result of the selection by Strategy 1 for this flight is computed from the meet-time trajectory with an FPA of $-3.4^{\circ}$. This fuel burn is generally higher than that of the minimum-fuel trajectory with an FPA of $-2.8^{\circ}$. Now suppose Strategy 2 with an $\gamma_{0}$ of $-2.3^{\circ}$ is used. While the selected FPA must be from one of the meet-time trajectories, the relationship between the descent CAS and the FPA of the trajectory must satisfy the FPA function defined in Eq. 1. This FPA function is represented by the solid vertical steps shown in Figure 5. The fuel burn as a result of the selection by Strategy 2 is computed from the trajectory that intercepts with the steps representing the FPA function. This trajectory has an FPA of $-2.6^{\circ}$. Hence all three strategies select distinct FPAs for this flight.

If no meet-time trajectories have the FPA defined by Strategy 1 or satisfy the FPA-descent-CAS relationship defined by Strategy 2, then a failure is recorded for this parameter of the strategy. The number of failures is used to determine whether the parameter should be rejected.

The meet-time trajectories labeled "STA2" in Figure 5 show an example of a flight with an STA2 later than STA1, in which the meet-time trajectories cross over the gap of the vertical steps defining the FPA function of strategy 2. This case is still considered a "success," and the vertical step with a closer achievable time-to-fly to the metering fix is selected as the FPA.

\section{V.F. Simulation and Data Analysis}

A fast-time Monte Carlo simulation was performed to generate the meet-time trajectories for small jet arrivals to DFW. For each arrival flight, a delay time and a descent weight were modeled from the distributions described in Sections V.D and V.C. The meet-time algorithm computed a set of fixed-FPA meet-time trajectories for the test condition specified, using the RUC data in the TS calculation of the trajectories. The fuel burn and speed-brake usage were recorded for further analysis to be described below. All data were categorized by gates and days.

Analysis of the meet-time trajectories for all the flights achieves both parts of the methodology described in Section V.A. The following paragraphs detail how the first part of the methodology, which is selection 
of the parameters, was achieved. The analysis selects $\gamma_{\text {univ }}$ for Strategy 1 from values between $-1.8^{\circ}$ and $-5.5^{\circ}$. The analysis selects $\gamma_{0}$ for Strategy 2 from $-1.8^{\circ}$ to $-3.7^{\circ}$. For each $\gamma_{\text {univ }}$ and $\gamma_{0}$, the average fuel burn per flight and feasibility rate are computed for all flights. The feasibility rate is the ratio of the flights with flyable FPAs (total number of "success") to the total flights analyzed. It must be $99 \%$ or better for $\gamma_{\text {univ }}$ or $\gamma_{0}$ to be selected (see Section III.E). Figure 6 sketches a notional average fuel burn per flight and feasibility rate as a function of $\gamma_{\text {univ }}$ or $\gamma_{0}$. The $\gamma_{\text {univ }}$ or $\gamma_{0}$ that yields the least average fuel burn per flight while having a feasibility rate of at least $99 \%$ is selected. This figure shows the dramatic effect the

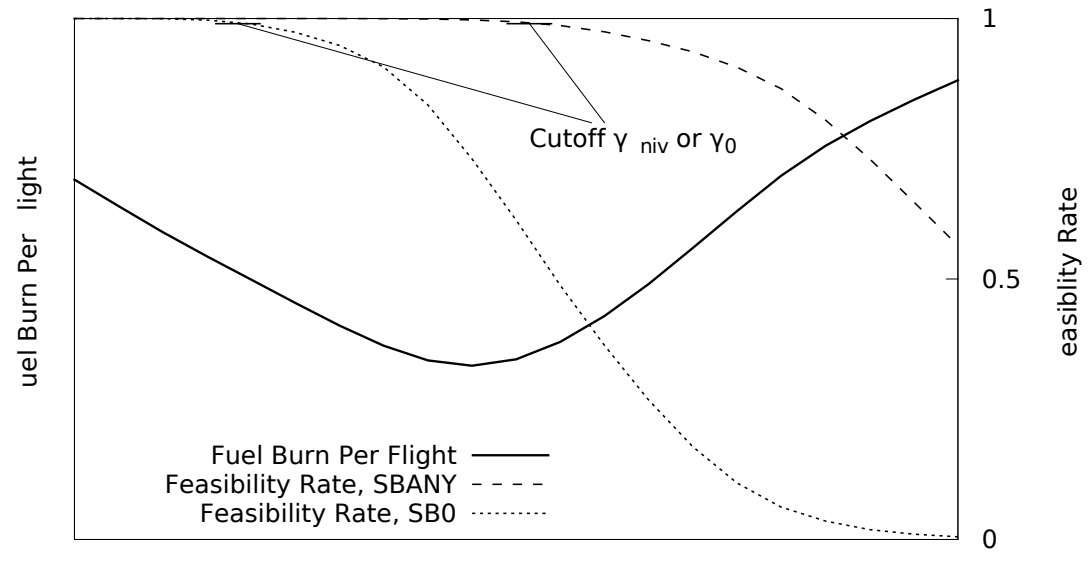

Yuniv or $Y_{0}$

Figure 6. Notional representation of the average fuel burn and feasibility rate. The feasibility rate falls off at steeper values of $\gamma_{\text {univ }}$ or $\gamma_{0}$.

planned-speed-brake usage on the feasibility rate and the selection of the parameters. Recall that two levels of planned speed-brake usage were described in Section III.D. Here Speed-Brake-Any (SBANY) allows any FPA to be considered for a flight as long as the modeled speed-brake usage is within the speed-brake capacity for that aircraft. The lenient SBANY condition very often allows the minimum-fuel FPA to be selected, as the computed cutoff $\gamma_{\text {univ }}$ or $\gamma_{0}$ (using the criterion of $99 \%$ feasibility rate) for SBANY is frequently steeper than the minimum-fuel $\gamma_{\text {univ }}$ or $\gamma_{0}$. Speed-Brake-Zero (SB0) represents the case that FPAs for a flight are limited to those that require no speed-brake usage. The SB0 condition has a steeper cutoff FPA that would restrict the acceptable $\gamma_{\text {univ }}$ or $\gamma_{0}$ to shallower, less fuel-efficient values.

The preceding selection criteria were used to select $\gamma_{\text {univ }}$ and $\gamma_{0}$ for each of the eight adaptation types listed in Section IV.C. For the Airport-Static adaptation, all flights were analyzed for the selection. For the other adaptations, a subset of the flights was analyzed to select $\gamma_{\text {univ }}$ or $\gamma_{0}$ for a gate and/or a timespan. For example, a total of sixteen pairs of $\gamma_{\text {univ }}$ and $\gamma_{0}$ were selected for the Gate-Season adaptation (four gates times four seasons), each using the flights into a specific gate during a specific season.

Selection of the parameters based on the feasibility rate ensures that the vast majority of flights will have flyable FPAs, but it does not consider the variation of winds that can make the flights through some gates on some days particularly difficult to fly. To ensure the feasibility rate for any given day and gate is "tolerable," a feasibility rate of $80 \%$ or better for any pair of gate and day is required for all adaptations.

For achieving the second part of the methodology, the fuel-burn benefits of trajectories by applying the three strategies are compared. For each flight, a fuel-burn penalty associated with a selected FPA is defined as the extra fuel burn of the trajectory with the selected FPA with respect to the fuel burn of the trajectory with the the minimum-fuel FPA. By definition, Strategy 3 results in zero fuel-burn penalty. The fuel-burn penalties for the FPAs selected by Strategy 1 and Strategy 2 are computed for all flights as a metric for the difference of fuel-burn benefits between the three strategies.

\section{Results}

Results of the fast-time simulation are presented as follows: Section VI.A summarizes the statistics of the number and aircraft types of the arrival flights used in the simulation. Section VI.B shows the selected 
FPAs for flights using Strategy 3 and discusses their correlation with winds. Sections VI.C and VI.D show the selected FPA and FPA function for Strategy 1 and Strategy 2, respectively, in eight adaptation types and two levels of planned speed-brake usage. Section VI.E compares the fuel-burn benefits of the three strategies.

\section{VI.A. Arrival Flights}

Table 4 shows the total number of arrival flights of small jets identified and used in this analysis. Due

Table 4. Total number of arrival flights of small jets in the analysis

\begin{tabular}{rrrrrrr}
\hline Month & Days & NE & SE & NW & SW & Missing Days \\
\hline Jan & 28 & 3322 & 2515 & 1204 & 1133 & $1,19,24$ \\
Feb & 21 & 2449 & 1761 & 971 & 909 & $11,16,18-20,23,25$ \\
Mar & 30 & 3629 & 2644 & 1474 & 1265 & 6 \\
Apr & 30 & 2963 & 2686 & 1614 & 1468 & 21,22 \\
May & 29 & 2908 & 2456 & 1428 & 1265 & 16,23 \\
Jun & 30 & 3465 & 2615 & 1815 & 1393 & $21,27,28$ \\
Jul & 29 & 2908 & 2311 & 1791 & 1189 & \\
Aug & 28 & 2823 & 2404 & 1772 & 1213 & \\
Sep & 30 & 3256 & 2471 & 1643 & 1313 & \\
Oct & 31 & 3422 & 2465 & 1570 & 1409 & \\
Nov & 30 & 3424 & 2555 & 1605 & 1454 & \\
Dec & 31 & 3335 & 2620 & 1628 & 1399 & \\
\hline Total & 347 & 37904 & 29503 & 18515 & 15410 & \\
\hline
\end{tabular}

to occasional periods when the data feed was unavailable, the track data was missing for 18 of the 365 days. Among the days where the track data was available, some data were not recorded due to short interruptions of the data feed. The NE gate was the busiest gate, accounting for $38 \%$ of the arrival flights.

Figure 7 shows the most frequent small jet aircraft types observed among the arrival flights. The Embraer ERJ 145 and 135 accounted for more than $63 \%$ of the fleet, with the other 70 aircraft types making up the rest.

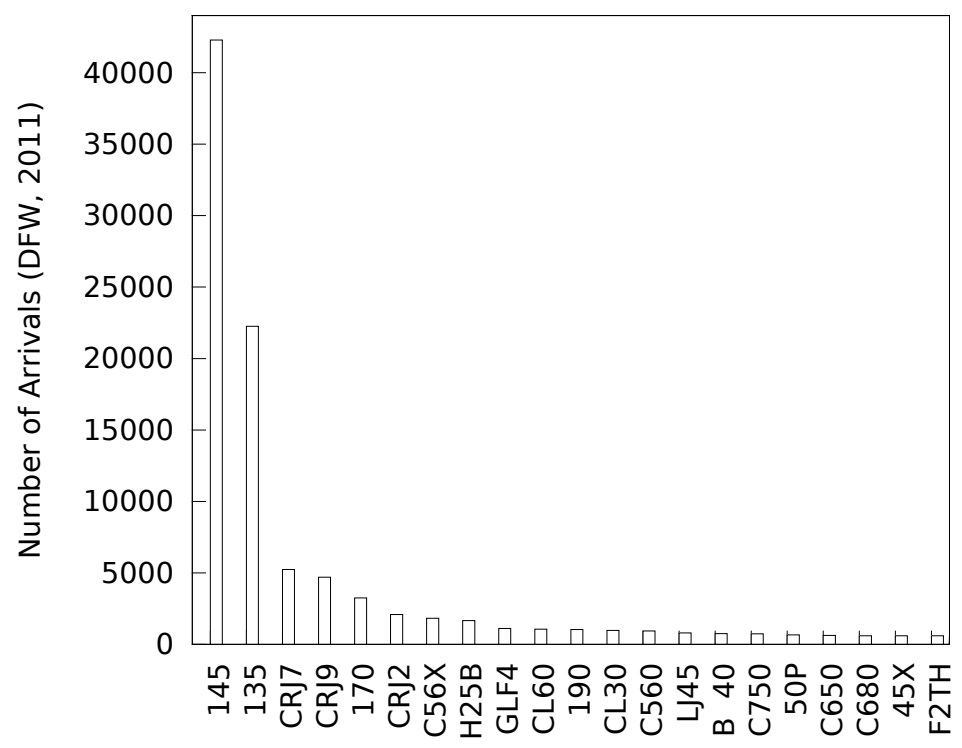

Figure 7. Most frequent arrival small jet types observed in Dallas/Fort Worth Airport, 2011. 


\section{VI.B. Strategy 3}

Strategy 3 selects a minimum-fuel FPA for each flight. Figure 8 shows the distribution of the FPAs selected for all flights under the SBANY condition. In the first four and last two months, steeper FPAs

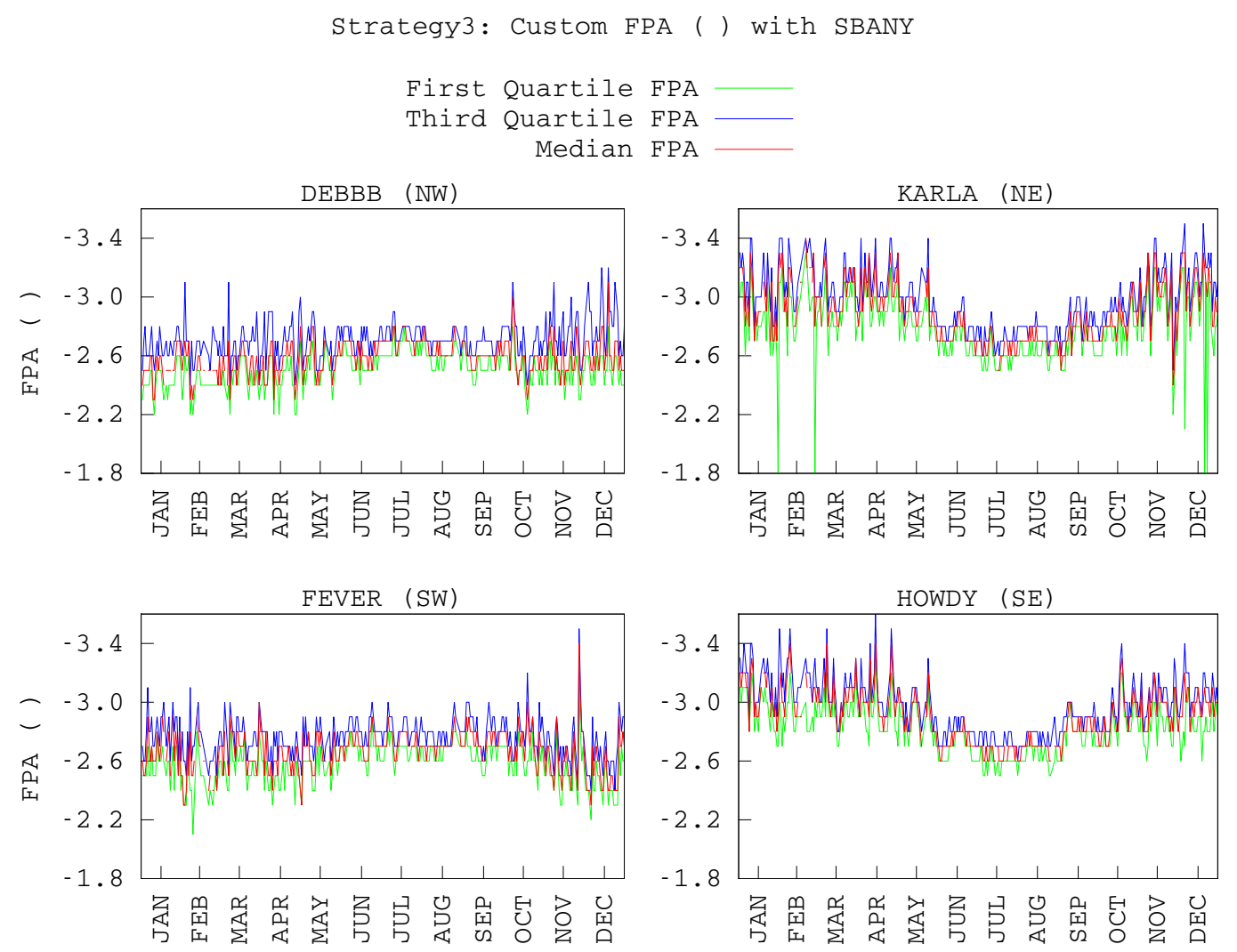

Figure 8. The first quartile, third quartile, and median FPAs selected using Strategy 3 under the SBANY condition.

are selected for KARLA and HOWDY while shallower FPAs are selected for DEBBB and FEVER. This is expected since DEBBB and FEVER arrivals experienced mostly tailwinds in these months, while flights entering KARLA and HOWDY experienced mostly headwinds. In these months, both larger fluctuations of the FPAs across days and wider variation of FPAs among flights within a day were observed. This is due to stronger magnitudes and variations of winds, and is consistent with Figure 3. For KARLA (NE), the third quartile FPA were as steep as $-3.5^{\circ}$ on Dec. 6th and 20th. For HOWDY (SE), the third quartile of the selected FPAs reached the steepest $-3.6^{\circ}$ on Apr. 15th. In July and August, the FPAs selected for all four gates are close to $-2.6^{\circ}$ and $-2.7^{\circ}$, implying weaker winds with more or less uniformly distributed directions. This observation is again consistent with Figure 3. The direction and magnitude of wind is apparently the strongest discriminator, causing correlated fluctuations of the selected FPAs for all four gates.

Although steeper FPAs are typically selected for strong headwinds, a few exceptions occurred, as shown for KARLA on Jan. 31st, Feb. 28th, Dec. 21th, and Dec. 23th, where the first quartile FPA was found to be $-1.8^{\circ}$. In fact, inspection of the data on Feb. 28th revealed that the shallowest FPA of $-1.8^{\circ}$ was selected for more than $45 \%$ of the flights (not shown in figure). This suggests that very shallow FPAs may be the most fuel-efficient in some conditions of strong headwinds, and will be further investigated in Section VII. In contrast, the shallow first quartile FPA of $-2.2^{\circ}$ observed for KARLA on Nov. 27th, nonetheless, is the result of a unusual tailwind on that day. The tailwind corresponds to a headwind for FEVER and resulted in the third quartile FPA peaking at $-3.5^{\circ}$. The shallow first quartile FPA of $-2.1^{\circ}$ observed for KARLA on Dec. 6th was possibly a mixed case between the exceptions and the normal days, and was not further investigated.

Figure 9 presents distributions of the FPAs selected for flights using Strategy 3 under the SB0 condition. In general, the values in Figure 9 are very close to those in Figure 8. As expected from SB0, some values of FPAs are shallower by $0.1^{\circ}$ and occasionally $0.2^{\circ}$, because the use of the speed-brake drag is forbidden during 


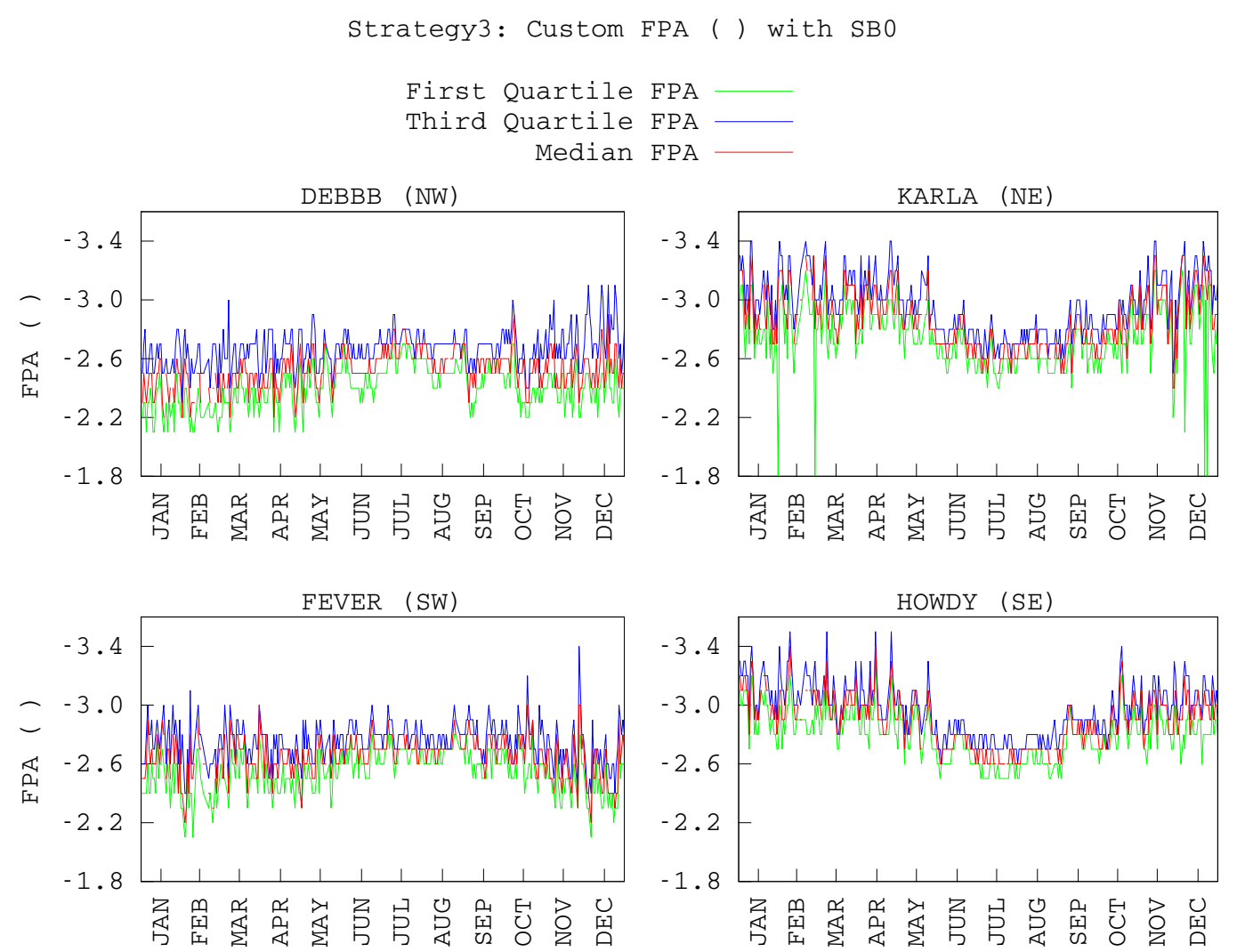

Figure 9. The first quartile, third quartile, and median FPAs selected using Strategy 3 under the SB0 condition.

descent. These results suggest that the SB0 condition has little impact on the selection of the minimum-fuel FPA on a flight by flight basis.

\section{VI.C. Strategy 1}

Strategy 1 selects the FPA based on the average fuel burn per flight and the feasibility rate. Although the feasibility rate falls off with steeper FPAs, for SBANY the FPAs satisfying the feasibility rate criterion usually allows the minimum-fuel FPA and even steeper values. Therefore, the fuel-burn criterion is the limiting factor for SBANY.

Figure 10 shows the values of $\gamma_{\text {univ }}$ selected for the airport-specific adaptations of Strategy 1 . In general, the $\gamma_{\text {univ }}$ selected for an adaptation of shorter timespan fluctuates mostly at or above the $\gamma_{\text {univ }}$ selected for a longer timespan. For the $\gamma_{\text {univ }}$ selected for the Airport-Day adaptation, the fluctuation is larger in winter and smaller in summer. The SB0 condition had a much stronger effect on Strategy 1 than on Strategy 3. Results of the SB0 condition shown on the right hand side of Figure 10 had a huge impact on $\gamma_{\text {univ }}$, shifting $\gamma_{\text {univ }}$ towards the shallow end by $0.3^{\circ}$ to $0.4^{\circ}$. The impact of the SB0 condition is further discussed in Section VI.D.

Figure 11 shows values of $\gamma_{\text {univ }}$ selected for the gate-specific adaptations of Strategy 1 under the SBANY condition. A similar trend was observed in Figure 11 as in Figure 8. The values of Gate-Day FPA are close to the values of median FPA in Figure 8. Steeper values of $\gamma_{\text {univ }}$ for KARLA and HOWDY are observed in the first four and last two months. On some days, the $\gamma_{\text {univ }}$ selected for KARLA and HOWDY were as steep as $-3.4^{\circ}$. As observed for Strategy 3, the anomaly of $-1.8^{\circ}$ selected for KARLA on Feb. 28th was due to the fact that almost half of the flights on that day had $-1.8^{\circ}$ as their minimum-fuel FPA. This is investigated further in Sec. VII. Generally speaking, the selected values of $\gamma_{\text {univ }}$ for KARLA and HOWDY are very close, with HOWDY having slightly steeper values of $\gamma_{\text {univ }}$. The FPAs for DEBBB and FEVER are very close, with DEBBB having slightly shallower values of $\gamma_{\text {univ }}$. An unusually shallow $\gamma_{\text {univ }}$ of $-2.3^{\circ}$ was selected for KARLA on Nov. 27th. The other values of $\gamma_{\text {univ }}$ selected for HOWDY, FEVER, and DEBBB 
Strategy1 Adapted to Airport

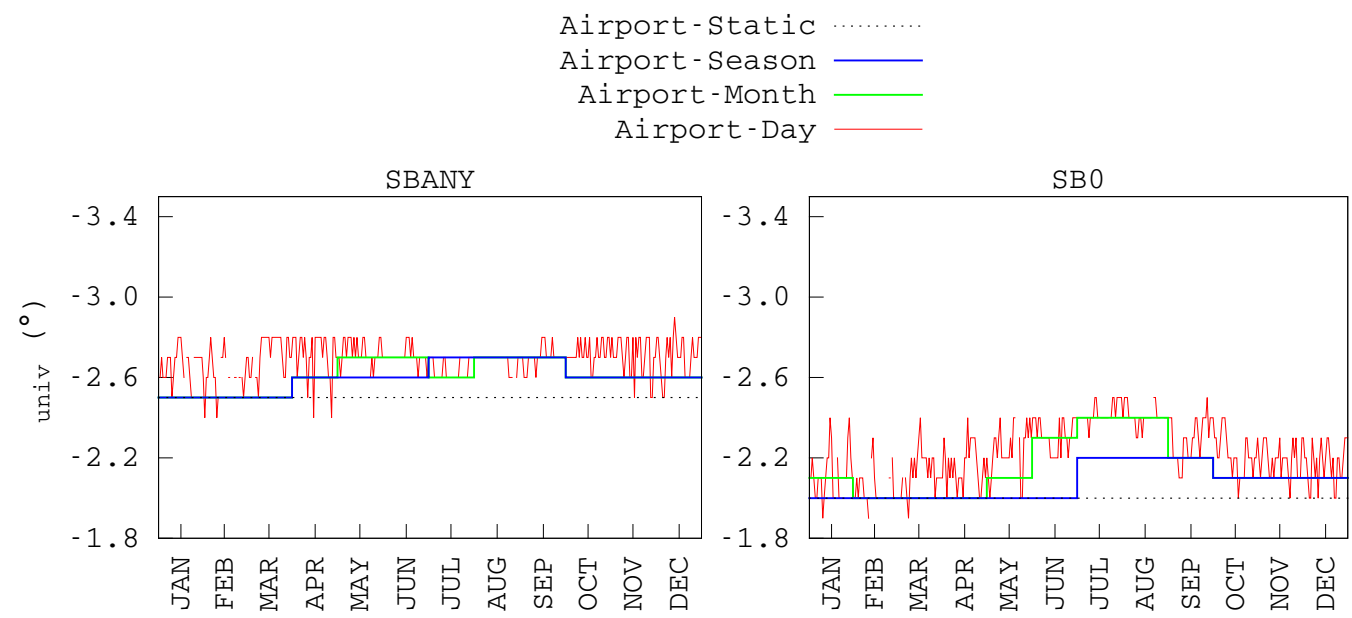

Figure 10. Values of $\gamma_{\text {univ }}$ selected for the airport-specific adaptations of Strategy 1.

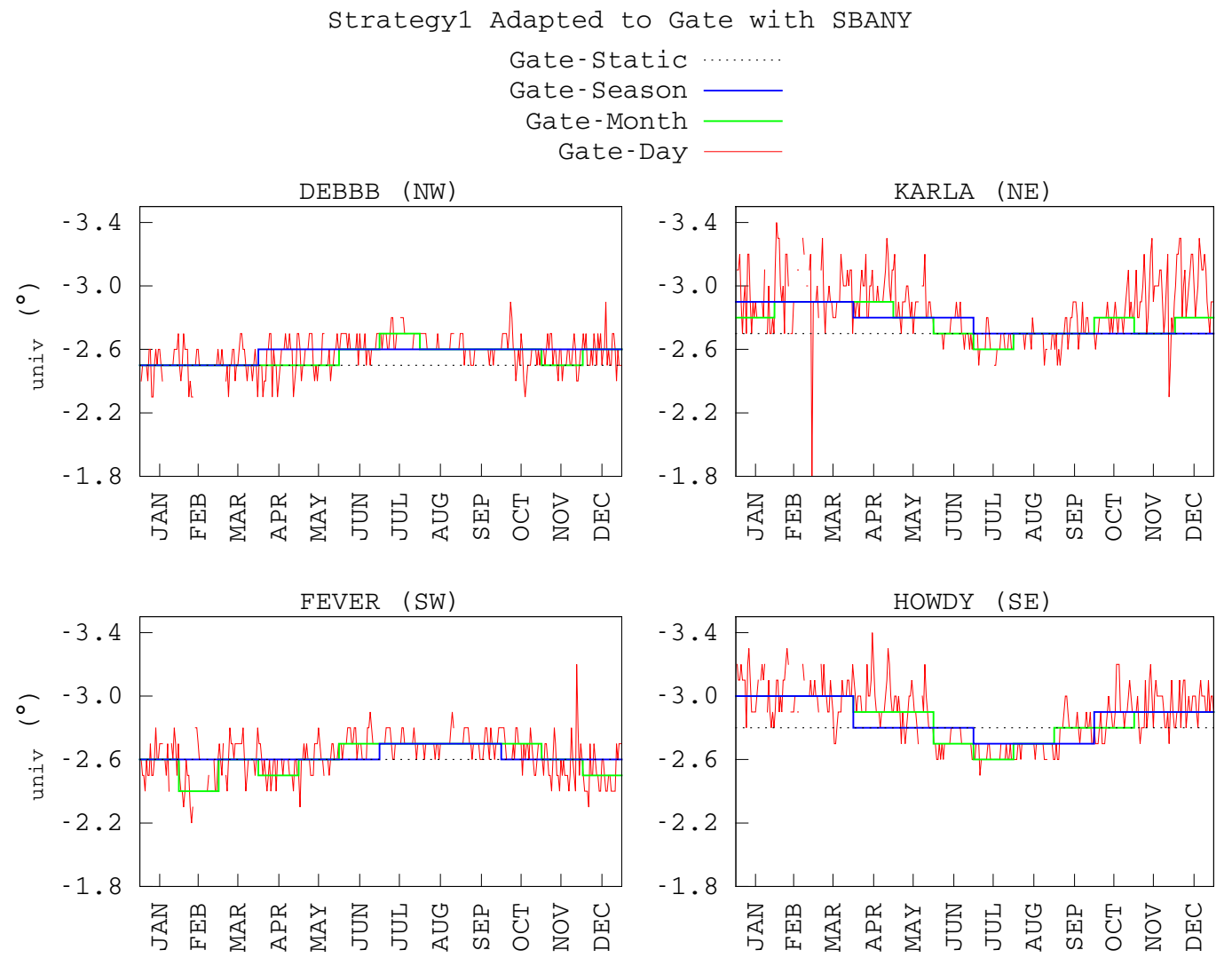

Figure 11. Values of $\gamma_{\text {univ }}$ selected for the gate-specific adaptations of Strategy 1 under the SBANY condition.

on this day are $-2.7^{\circ},-3.2^{\circ}$, and $-2.4^{\circ}$, respectively. These values suggest an average direction of the wind from the north. Inspection of the RUC data revealed a fairly strong wind of 85 knots from the NNE at altitudes between 20,000 ft and 25,000 ft. Similar values of FPA were observed on Nov. 28th and the wind again came from NNE, although the wind was strongest between 28,000 ft and 35,000 ft.

Figure 12 shows values of $\gamma_{\text {univ }}$ selected for the gate-specific adaptations of Strategy 1 under the SB0 
condition. The SB0 condition reduced the number of feasible trajectories for each flight and made the

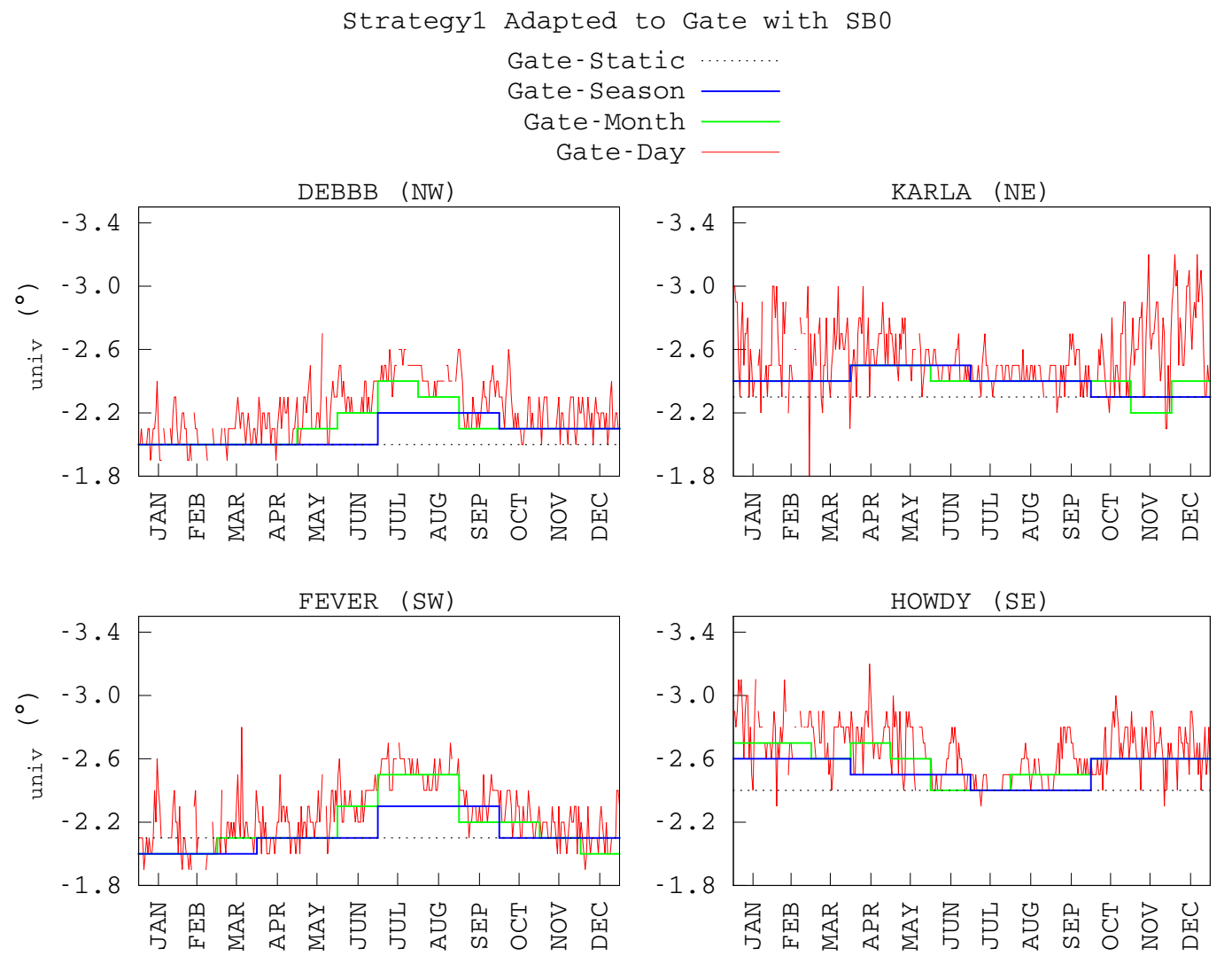

Figure 12. Values of $\gamma_{\text {univ }}$ selected for the Gate-Static, Gate-Season, Gate-Month, and Gate-Day adaptations of Strategy 1 under the SBO condition.

feasibility rate fall off. In contrast to the SBANY condition, the feasibility rate of $99 \%$ becomes the limiting factor instead of the fuel burn. The overall effect is a shift of $0.3^{\circ}$ to $0.4^{\circ}$ of the $\gamma_{\text {univ }}$ towards shallow values compared to Figure 11. The variation of $\gamma_{\text {univ }}$ from one gate to another reached $1.3^{\circ}$ on Apr. 15th, when $-3.2^{\circ}$ was selected for HOWDY and $-1.9^{\circ}$ was selected for DEBBB. The variation $\gamma_{\text {univ }}$ from one day to another reached $1.4^{\circ}$ for KARLA, when $-1.8^{\circ}$ was selected on Feb. 28th and $-3.2^{\circ}$ was selected on Nov. 14th.

Note that the values of $\gamma_{\text {univ }}$ for the airport-specific adaptations shown in Figure 10 are very close to the values of $\gamma_{\text {univ }}$ selected for DEBBB shown in Figures 11 and 12. This is because the selected $\gamma_{\text {univ }}$ must ensure a feasibility rate of $80 \%$ for any given gate on any day. Therefore, the $\gamma_{\text {univ }}$ selected for airport adaptations is "almost" completely constrained by the shallowest FPAs selected for DEBBB.

\section{VI.D. Strategy 2}

Recall that the family of FPA functions described in Section III changes the selected FPA by $0.1^{\circ}$ for every 10 knots of the descent CAS. A selected FPA function is defined by the FPA it yields at 250 knots, denoted as $\gamma_{0}$. Figure 13 shows $\gamma_{0}$ selected for the airport-specific adaptations of Strategy 2. Similar to the values of $\gamma_{\text {univ }}$ in Figure 10, values of $\gamma_{0}$ for an adaptation of shorter timespan fluctuate mostly at or above values of $\gamma_{0}$ selected for a longer timespan. For values of $\gamma_{0}$ selected for the Airport-Day adaptation, the fluctuation is larger in winter and smaller in summer. Results of the SB0 condition, shown on the right hand side of Figure 13, again had a huge impact on the selected FPA, shifting the values of $\gamma_{0}$ towards the shallow end by $0.3^{\circ}$ to $0.4^{\circ}$.

Figure 14 shows $\gamma_{0}$ selected for the gate-specific adaptations of Strategy 2 under the SBANY condition. The values of $\gamma_{0}$ are roughly shallower than the values of $\gamma_{\text {univ }}$ for Strategy 1 in Figure 11 by $0.1^{\circ}$. The trends are apparently similar between the two strategies. Observing the values of the parameter for the Gate-Static adaptation for these two strategies, the gates ordered in terms of the steepness of their FPAs are HOWDY, KARLA, FEVER, and DEBBB. 
Strategy2 Adapted to Airport

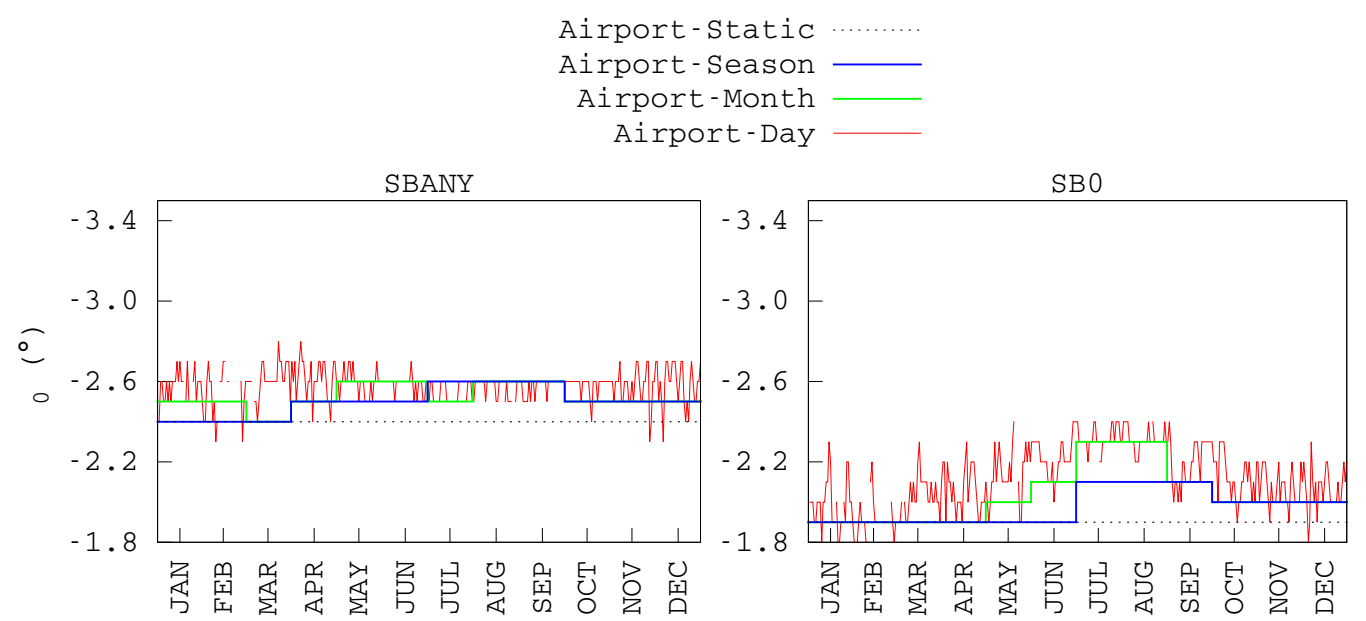

Figure 13. Values of $\gamma_{\text {univ }}$ selected for the airport-specific adaptations of Strategy 2.
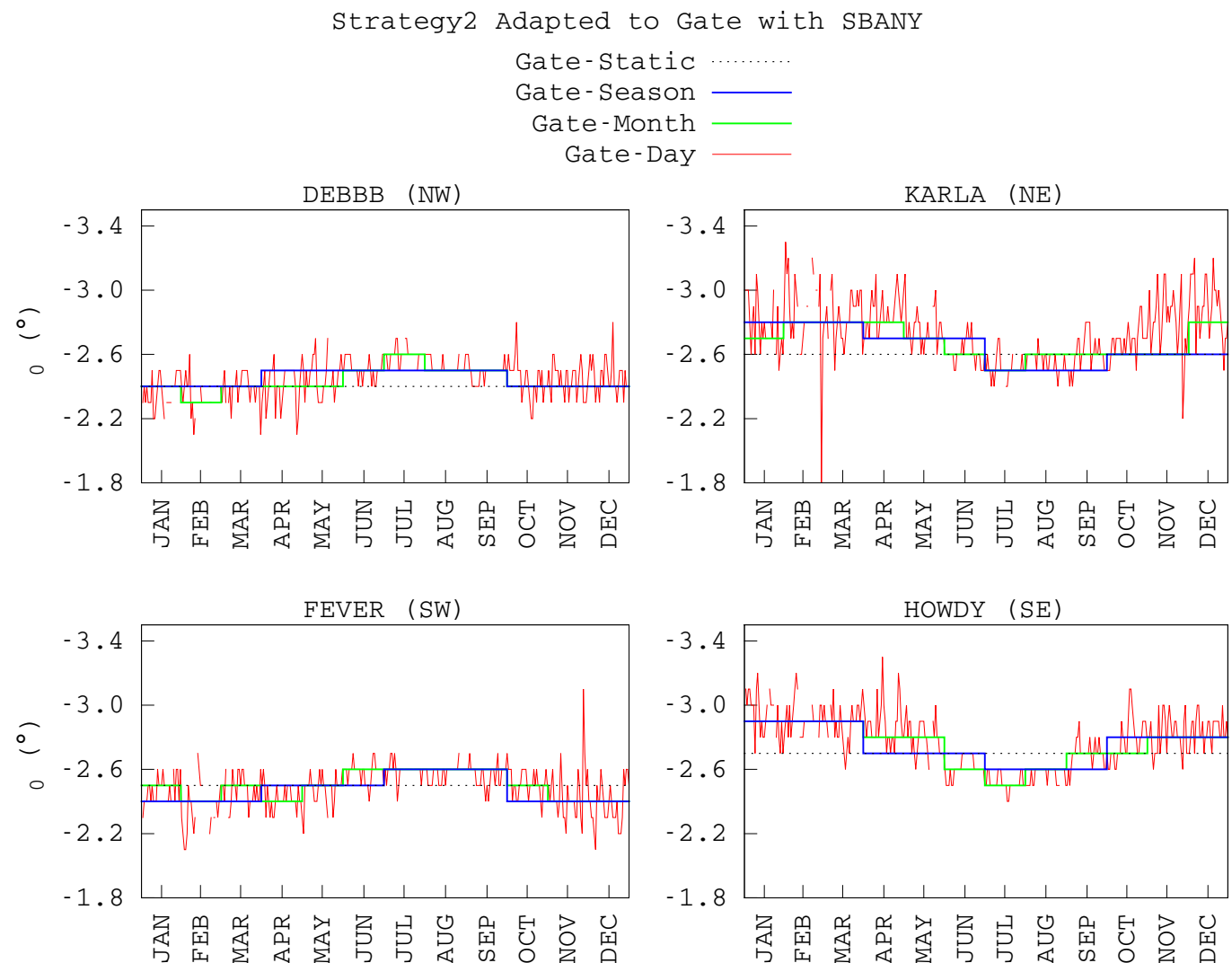

Figure 14. Values of $\gamma_{0}$ selected for the Gate-Static, Gate-Season, Gate-Month, and Gate-Day adaptations of Strategy 2 under the SBANY condition.

For the SB0 condition, Figure 15 shows values of $\gamma_{0}$ selected for the gate specific adaptations of Strategy 2. Similar to Strategy 1, the SB0 condition had a huge impact on the selected FPA function, shifting $\gamma_{0}$ to shallower values by $0.4^{\circ}$ to $0.5^{\circ}$. Similar to Strategy $1, \gamma_{0}$ selected for KARLA on Feb. 28th was $-1.8^{\circ}$ at 250 knots for both SBANY and SB0 conditions. Similar to Strategy 1, the selection of $\gamma_{0}$ in Figure 13 is constrained by the results for DEBBB in Figures 14 and 15 . Therefore the values of $\gamma_{0}$ in Figure 13 are very 

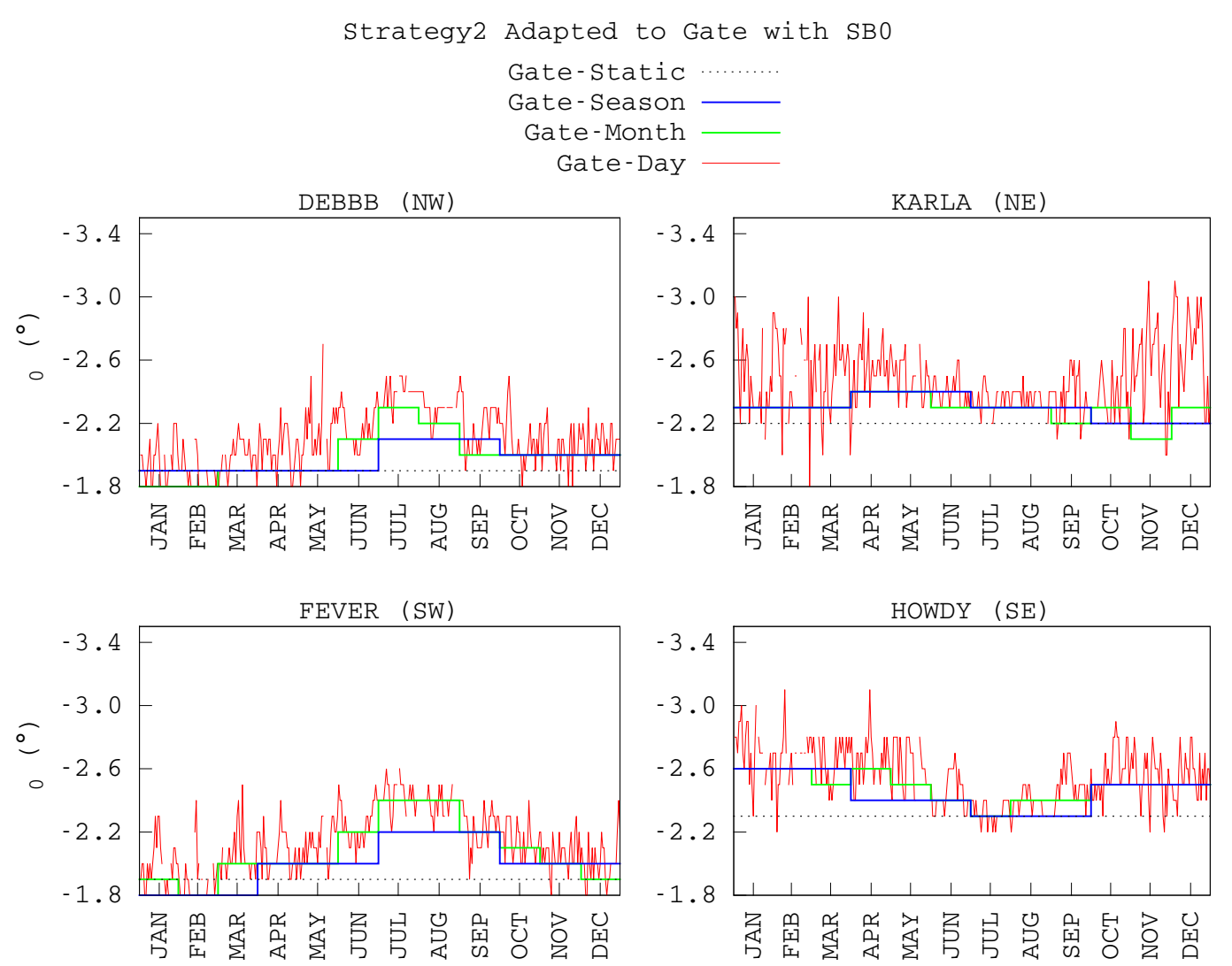

Figure 15. Values of $\gamma_{0}$ selected for the Gate-Static, Gate-Season, Gate-Month, and Gate-Day adaptations of Strategy 2 under the SB0 condition.

close to those in Figures 14 and 15.

\section{VI.E. Fuel Burn Comparison}

The culmination of the preceding analysis is the comparison of fuel consumption across the strategies and the adaptation types studied. While it would be interesting to estimate the actual fuel burn of flights under actual arrival-metering operations, it would not contribute to the choice of a FPA-defining strategy for EDAassisted CDA operations. Instead, the salient question is: "what FPA strategy would provide the best value for implementation?" To facilitate the comparison, results are presented in terms of the average fuel burn per flight, over the year's worth of DFW traffic data, relative to the minimum-fuel solution of Strategy 3. In this way, the results will show how close the simpler strategies (1 and 2), and their adaptations can come to the minimum-fuel solution without requiring real-time pilot-controller communication of FPA just prior to top of descent.

The fuel burn comparison was based on trajectories from the freeze horizon before the top-of-descent to the metering fix, and therefore has contributions from both the cruise and the descent segments. Figure 16 shows the fuel-burn penalty per flight computed for Strategies 1 and 2. Overall, two interesting observations can be made. First, even the simplest strategy, a single static FPA adapted for DFW, has the potential to come within $26 \mathrm{lbs}$ of the minimum-fuel solution for each flight on average. This $26 \mathrm{lbs}$ represents the potential benefit of the minimum-fuel (Strategy 3) solution. To put this into perspective, this represents approximately $5 \%$ of the typical small-jet arrival transitioning over a $160 \mathrm{nmi}$ segment from cruise to the TRACON boundary. This of course assumes that the modeled winds aloft and traffic conditions used for parameter selection are reasonably accurate.

The second observation relates to the potential impact of speed brake usage. While the planned routine use of speed brakes in such an arrival procedure is problematic from a flight operation's point of view, it is very interesting to note that the use of speed brakes has the potential to recover upwards of two-thirds of the difference in fuel burn between the minimum fuel solution and a static adaptation of Strategy 1 or 2 . 


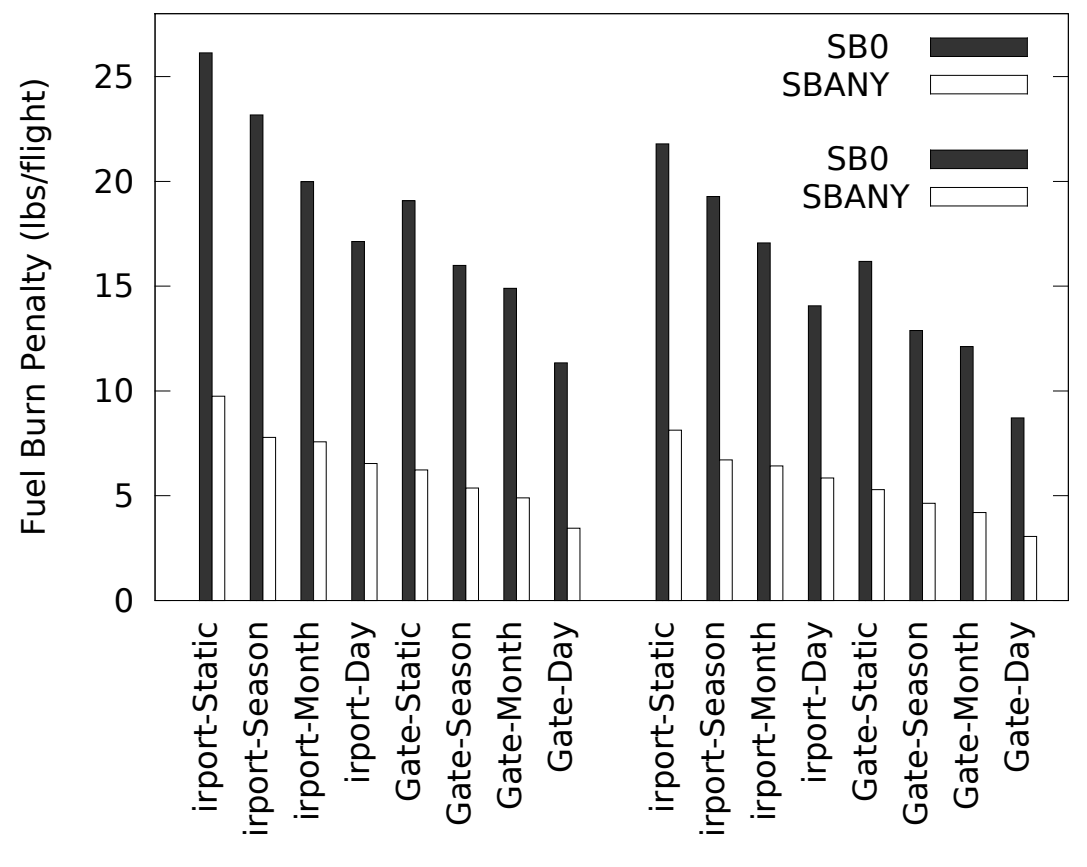

Strategy 1

Strategy 2

Figure 16. Fuel burn penalty calculated for various adaptations of Strategies 1 and 2 .

In other words, the planned use of up to the full speed brake drag modeled in this study (SBANY) is more effective at reducing fuel consumption than any of the studied adaptations of Strategy 1 or 2 under the SB0 condition. This highlights the sensitivity of the fuel burn to FPA limits in Strategies 1 and 2 stemming from strong wind conditions. Without the use of speed brakes, the strong tailwind conditions would otherwise prevent Strategy 1 or 2 from selecting FPAs that are far more fuel efficient during the rest of the year, month, or season. For the remainder of this section, only the SB0 condition (no planned use of speed brakes) will be discussed.

Returning to the primary focus of this paper, the potential impact of directional and temporal adaptations on the fuel efficiency of Strategies 1 and 2, consider the Strategy1 results on the left side of Figure 16. Relative to the Airport-Static adaptation, adaptation to arrival-gate direction has the potential to reduce the "extra" $26 \mathrm{lbs}$ of fuel per flight by $27 \%$. In other words, publishing a universal FPA for each of the four gates will recover a little more than a quarter of the way to the minimum fuel solution. By comparison, adapting the universal "airport" FPA to season, month and day has the potential of reducing that extra 26 lbs of fuel per flight by $11 \%, 24 \%$, and $34 \%$, respectively.

When combined, the directional and temporal adaptations together have the potential for reducing the $26 \mathrm{lbs}$ of extra fuel burn by $57 \%$. Essentially, the combined adaptation of a universally fixed FPA for each arrival gate, for each day of operations, recovers more than half of the fuel savings of the minimum-fuel solution for each flight.

In considering the overall results for Strategy 2 shown in Figure 16, both the directional and temporal adaptations yield similar improvements in fuel efficiency. By "adapting" to the descent speed, Strategy 2 is essentially a surrogate adaptation for metering delay, one of the primary factors being considered in this analysis. As such, this strategy was anticipated to yield a fair amount of benefit under metering conditions. The results indicate that Strategy 2 contributes a $17 \%$ reduction in the $26 \mathrm{lbs}$ of extra fuel burn over Strategy 1 for the Airport-Static case, and anywhere from 15-23\% depending on the extent of adaptation. The combined effect of Strategy 2 with both directional and temporal adaptation has the potential to achieve $67 \%$ of the minimum-fuel (Strategy 3 ) benefit. 


\section{Discussion}

\section{VII.A. A Very Shallow Minimum-Fuel FPA}

The selection of $-1.8^{\circ}$, the shallowest FPA analyzed, for Strategy 1 for KARLA (NE) on Feb. 28th raised questions as to what kind of headwinds can result in a fuel-efficient shallow FPA. Strong headwinds for the NE gate were observed on many days, but only the calculation for Feb. 28th showed $-1.8^{\circ}$ as the overall minimum-fuel FPA. To illustrate this "anomalous" behavior, the meet-time trajectories of two flights were compared below.

Figure 17 demonstrates the fuel burn of the meet-time trajectories for two E145 flights entering the NE gate on two distinct days. EGF3271 entered the NE gate on Feb. 1st and had a minimum-fuel FPA at

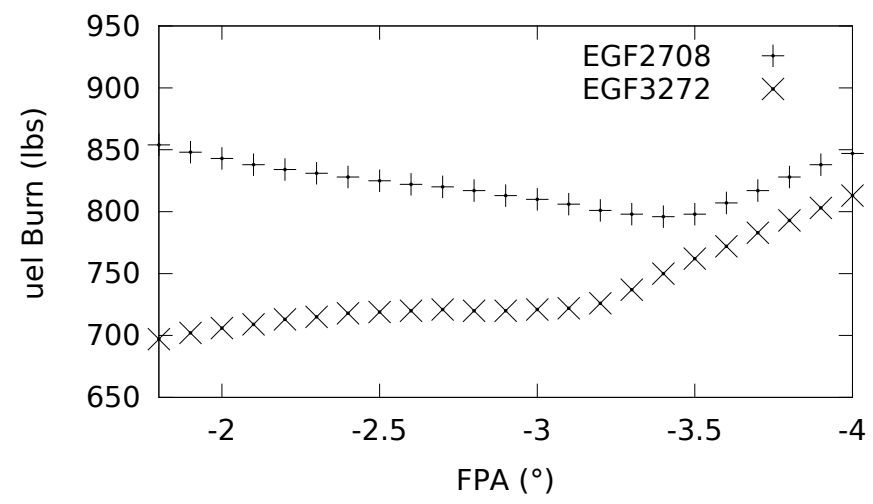

Figure 17. Fuel burn of the meet-time trajectories for two flights entering the NE gate. While EGF2708 had a minimum-fuel FPA of $-3.4^{\circ}$, EGF3272 had a minimum-fuel FPA of $-1.8^{\circ}$.

$-3.4^{\circ}$. Such minimum-fuel FPA in the middle of the range of sampled FPAs is characteristic of the meet-time trajectories of most arrival flights. Head winds typically shift the minimum-fuel FPA to a steeper value, while tailwinds usually shift the minimum-fuel FPA to a shallower value. However, a small fraction of flights in the presence of strong headwinds had an inversion of slope at shallow FPAs, causing $-1.8^{\circ}$ to be the most fuel-efficient of all FPAs. EGF3272 on Feb. 28th in Figure 17 was one of them. This inversion had been observed in previous work, ${ }^{8}$ and seemed to occur most frequently in the presence of strong headwinds.

Careful examination of the 114 flights entering the NE gate on Feb. 28th revealed 54 flights (45\%) that had $-1.8^{\circ}$ as their minimum-fuel FPA. The accumulated fuel-burn advantage of $-1.8^{\circ}$ in the 54 flights overpowered the fuel-burn disadvantage in the remaining 60 of the 114 flights and resulted in the selection of $-1.8^{\circ}$ as the overall minimum-fuel FPA. A detailed examination of flights of the entire year entering the NE gate showed about 1200 flights that had $-1.8^{\circ}$ as their minimum-fuel FPA. The headwinds at their cruise altitudes were typically strong, although mild headwinds and even mild tailwinds were observed for some flights. The results suggest that a strong headwind promotes, but is not a necessary condition for, the inversion of slope. To look for special characteristics of the wind on Feb. 28th, the wind along the route of each flight entering the NE gate on Feb. 28th was further analyzed. It was observed that, for many of the 54 flights that had an inversion, the headwind weakened significantly along the descent segment. Therefore, it was possible that the inversion of slope was highly correlated with the wind gradient with respect to the altitude. Take the two flights shown in Figure 17 as an example. The headwind experienced by EGF2708 decreased from 110 knots at $36,000 \mathrm{ft}$ to 90 knots at 25,000 ft, while the headwind experienced by EGF3272 decreased from 120 knots at $36,000 \mathrm{ft}$ to 60 knots at 25,000 ft. The faster decay of the headwind for EGF3272 might contribute to favoring an early descent.

To support the above conjecture, the wind gradients were examined systematically, using a measure of the change of along-the-route wind experienced by a flight defined by Eq. 3:

$$
W_{i}^{\prime}=\frac{\left(W\left(x_{i}, y_{i}, z_{i}, t_{i}, \Psi_{i}\right)-W\left(x_{i}, y_{i}, z_{i}-\Delta z, t_{i}, \Psi_{i}\right)\right)}{\Delta z},
$$

where the subscript $i$ denotes the index of the flight, $W^{\prime}$ represents a measure of the change of the alongthe-route wind with respect to altitude, $x, y, z, t$ represent the flight's initial $4 \mathrm{D}$ position for the analysis, $\Psi$ represents the flight's heading, and $\Delta z$ represents a characteristic change of altitude. A value of $8,000 \mathrm{ft}$ was 
chosen for $\Delta z$. Calculation of $W_{i}^{\prime}$ for all flights entering the NE gate showed that, on average, the flights on Feb. 28th experienced the greatest change of the along-the-route wind. The average value of $W^{\prime}$ for the flights entering the NE gate was 4.4 knots per 1,000 ft. Feb. 27th had the second highest average of $W^{\prime}$ of 3.6 knots per 1,000 ft. Among the 125 flights entering the NE gate on Feb. 27th, 31 of them had $-1.8^{\circ}$ as their minimum-fuel FPA while the remaining 94 flights had other minimum-fuel FPAs. However, the fuel-burn advantages of $-1.8^{\circ}$ for these 31 flights were not enough to make $-1.8^{\circ}$ the overall minimum-fuel FPA for all flights. The other days had smaller $W^{\prime}$ and smaller fractions of flights with selected minimum-fuel FPAs of $-1.8^{\circ}$.

Although the results showed strong correlation between $W^{\prime}$ and the selection of a shallow FPA, $W^{\prime}$ was by no means the only parameter that could have resulted in a fuel-efficient shallow FPA. In fact, a small fraction of flights that had $-1.8^{\circ}$ as their minimum-fuel FPA had very small $W_{i}^{\prime}$. Future detailed analysis needs to better identify the combination of parameters that promote a shallow minimum-fuel FPA.

\section{VII.B. The Form of the FPA Function}

The speed-dependent FPA function in Strategy 2 was intended to account for the effect of the descent speed on the minimum-fuel FPA. However, for some gates on certain days, the selected FPA function resulted in more predicted fuel burn per flight than a universal FPA. The following discussion contrasts the relative fuel benefits of Strategies 1 and 2 with two examples. The results were based on the SBANY condition, although results of the SB0 condition followed a similar pattern. Figure 18 shows a specific gate-day pair for which Strategy 2 worked relatively well. The average fuel-burn penalties of the meet-time trajectories of the flights entering the SW gate on Feb. 28th under the SBANY condition were shown using a color map. Due to the fact that the metering delay, chosen from a uniform distribution, maps to a distribution denser

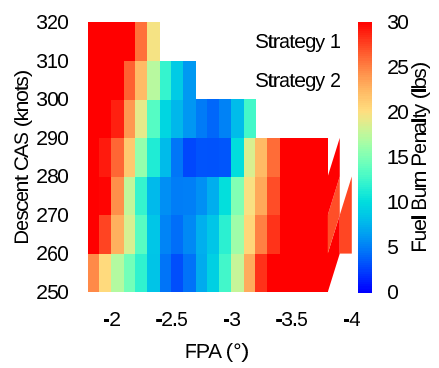

Figure 18. Fuel-burn penalty for the meet-time trajectories entering FEVER (SW) on Feb. 28th under the SBANY condition. 
in the low end of the descent CAS, trajectories were denser in the low descent CAS region than the high descent CAS region. No trajectories with FPAs steeper than $-4.0^{\circ}$ were found in Figure 18 because those trajectories all exceeded the speed-brake drag capacity. The "valley" area shifts roughly by $0.1^{\circ}$ for every 10 knots of the descent CAS, making it possible to "pick up" minimum-fuel trajectories with low fuel burn with the vertical steps of an FPA function illustrated in Figure 5. The resulting fuel-burn penalty is $4.1 \mathrm{lbs}$ with a selected $\gamma_{0}$ of $-2.4^{\circ}$. The fuel-burn penalty of applying Strategy 1 is a less favorable 7.4 lbs with a selected $\gamma_{\text {univ }}$ of $-2.4^{\circ}$. On the other hand, Strategy 2 did not work as well as Strategy 1 for the flights entering KARLA on Feb. 28th, as shown in Figure 19. It can be observed that a universal FPA of $-1.8^{\circ}$

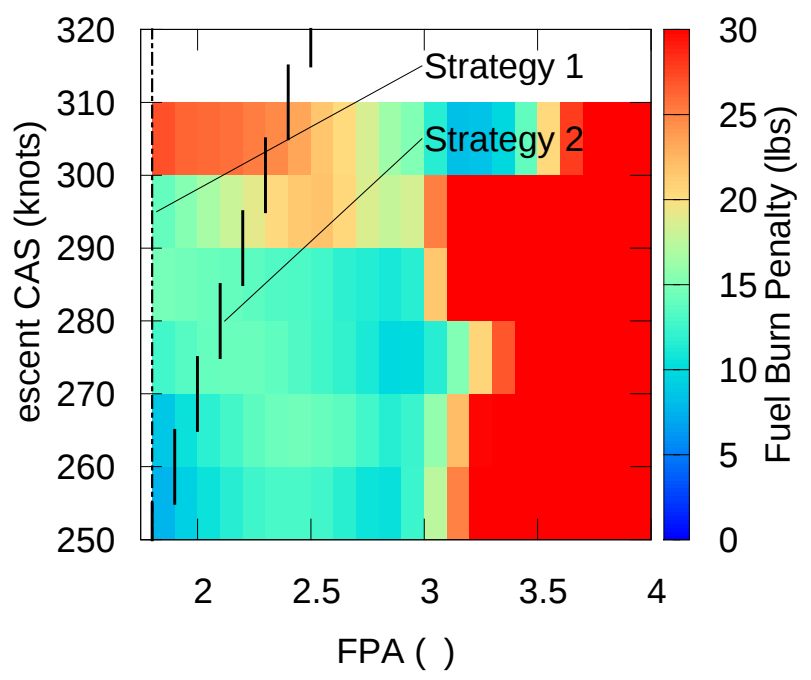

Figure 19. Fuel-burn penalty for the meet-time trajectories entering KARLA (NE) on Feb. 28th under the SBANY condition.

would pick up the trajectories with slightly lower fuel-burn penalty on the left vertical line of the figure. An FPA function must pick up those trajectories in the middle that have higher fuel-burn penalty than those on the left, therefore resulting in higher overall fuel burn penalty. The selected $\gamma_{0}$ is $-1.8^{\circ}$, and the associated fuel-burn penalty is $9.6 \mathrm{lbs}$, compared to a slightly lower fuel-burn penalty of $9.0 \mathrm{lbs}$ for a $\gamma_{\text {univ }}$ of $-1.8^{\circ}$ selected for Strategy 1.

The function form for Strategy 2 can potentially be extended to have as a second parameter the ratio of the change of DCAS and the change of FPA. Both $\gamma_{0}$ and this ratio must be selected via the methodology described in Section V.A. With this more general definition of the FPA function, Strategy 1 becomes a special case of Strategy 2 with a ratio of infinity.

\section{VII.C. Further Adaptation to Routes}

Given the wide range of arrival-route courses feeding a typical arrival gate, there is potential for additional benefit from adapting to specific arrival routes. Figure 2 clearly shows that variation of the ground course for each gate can be as wide as $90^{\circ}$ (see DEBBB, for example). Therefore, individual flights can experience very different wind along their routes even if they enter the same gate at roughly the same time. Particularly for the SB0 condition, few flights in tailwind would shift the selected FPA or FPA function to the shallow end. With adaptation to the routes, different FPAs or FPA functions are selected for different branches of the Standard Terminal Arrival Route. This adaptation is expected to further improve fuel-efficiency.

\section{VII.D. Implementation of the Strategies}

Looking towards the operational implementation of continuous descents under metering conditions, the Federal Aviation Administration (FAA) will have to decide upon an approach for defining continuous descent 
FPAs for small jets. While this paper compares and contrasts the relative fuel efficiency of candidate strategies for defining descent FPAs, other factors will enter the implementation decision, not the least of which will be the complexity and cost of implementing and supporting an FPA procedure.

Of the three strategies proposed and analyzed in this paper, Strategy 3 saves the most fuel but is the most costly and complex to implement, due to the requirement of communicating the FPA to the pilot in real time. Strategies 1 and 2 are both simple enough to lend themselves to off-line analysis to select their parameters, publication and dissemination before flight. Strategy 1 has the advantage of defining a single descent FPA for an airport. While appealing in its simplicity, this limits the potential fuel efficiency gained from adjusting the descent profile for operational factors such as speed and prevailing winds. Strategy 2, while slightly more complex in terms of defining FPA as a function of descent speed, has the potential to capture much of the fuel efficiency related to the descent speed. Both strategies lend themselves to fast-time analysis that can support the selection and publication of their parameters a day or more before flight. This would allow the FAA to adapt the appropriate parameters to each airport and disseminate those parameters through the aeronautical information network. Depending on the time horizon chosen for the adaptation (annual, seasonal, monthly, weekly or daily), this information may be made available in several ways. For longer periods of time, the parameters could be included as part of published arrival procedures or flight manual amendments. Alternatively, this information could be provided to pilots as part of their standard pre-flight planning and weather briefing. At the very least, this dissemination approach would be necessary for adaptations performed on a more frequent (e.g., daily) basis.

Perhaps the most critical implementation aspect associated with Strategies 1 and 2 is the adaptation of the FPA parameter(s) to specific airports and time horizons. Given the sensitivity of the selected FPA to prevailing winds and the lack of precise wind forecast for longer time horizons, analysis based on historical data or climatological data would lend itself to parameter adaptation on an annual, seasonal or monthly basis. Historical data from previous years may be used for such prediction, assuming a similar pattern of weather and wind. However, the validity of such an assumption remains to be verified. Such uncertainties would raise the lowest achievable fuel-burn penalty of the static, per-season, and per-month adaptation. For shorter adaptation horizons, on the order of daily updates, numerical weather prediction such as the RUC weather forecast would provide precise and relatively accurate forecasts. This would not only improve the fuel efficiency of the FPA or FPA-function adaptation, it would also provide the precision needed to support the adaptation of the FPA or FPA function to specific arrival routes feeding an arrival gate.

\section{Conclusion}

This paper proposed three strategies for choosing the descent flight-path angles (FPAs) for small jets in transition airspace under metering conditions. The three FPA selection strategies are:

1. Universally fixed FPA - defines a universal FPA for all small jets arriving to an airport, gate, or route.

2. FPA as a function of descent speed - defines different FPAs for different descent speeds.

3. Minimum-fuel FPA - computes minimum-fuel FPA for each flight, but requires communication of the FPA to the pilot in real time.

The three strategies vary in operational complexity. The minimum-fuel FPA strategy served as the reference point for the fuel-burn benefits. A full year's worth of traffic data arriving at the Dallas/Fort Worth (DFW) airport during 2011 was used for the analysis of the three strategies. The FPA of Strategy 1 and the FPA function of Strategy 2 were selected based on fuel burn and planned speed-brake usage of the meet-time trajectories computed for the flights. Two levels of planned speed-brake usage were analyzed to explore the impact of speed-brake usage on the selected FPA, FPA function, and the resulting fuel burn.

Results showed that the universally fixed FPA adapted for the DFW Airport had only 26 lbs of extra fuel burn relative to the minimum-fuel solution for each flight on average. To improve fuel-efficiency by reducing wind variation along the route, adaptations of Strategies 1 and 2 to the airport, the arrival gate (direction), and various timespans, were defined and analyzed. The adapted FPAs vary significantly, up to $1.3^{\circ}$ from one arrival gate to another, and up to $1.4^{\circ}$ from one day to another. Adaptation to the arrival-gate reduced the extra fuel burn per flight by up to $27 \%$. Adaptation to the day alone reduced the extra fuel burn per flight by up to $34 \%$. Combining the directional and temporal adaptations reduced the extra fuel burn by $57 \%$. The FPA function strategy, considered as a surrogate adaptation to the descent speed, reduced the 
extra fuel burn per flight by $17 \%$. The combined effect of the directional, temporal, and speed adaptation recovered $67 \%$ of extra fuel burn relative to the minimum-fuel solution.

In conclusion, the simple forms of the universally-fixed FPA Strategy and FPA function Strategy, together with the fact that they do not require explicit ground-air communication of the FPA in real time, make them favorable for implementation. Adaptations to direction and timespans reduced up to two-thirds of the extra fuel burn relative to the minimum-fuel solution. For future work, it would be interesting to analyze the adaptations of these strategies to other airports and/or routes. It would also be interesting to analyze the variation of winds with respect to years, which would help determine if historical data can provide adequate estimates of the winds for longer time horizons for the purpose of selecting FPAs.

\section{Acknowledgments}

We thank Richard Coppenbarger and Harry Swenson for helpful discussions.

\section{References}

1 "FAA's NextGen Implementation Plan," Federal Aviation Administration, March 2011.

2 "Concept of Operations for the Next Generation Air Transportation System," Joint Planning and Development Office (JPDO), Oct. 2009.

${ }^{3}$ Coppenbarger, R. A., Mead, R. W., and Sweet, D. N., "Field Evaluation of the Tailored Arrivals Concept for DatalinkEnabled Continuous Descent Approach," Journal of Aircraft, Vol. 46, No. 4, July-August 2009, pp. 1200-1209.

${ }^{4}$ Novak, D., Bucak, T., and Radišić, T., "Development, Design and Flight Test Evaluation of Continuous Descent Approach Procedure in FIR Zagreb," PROMET - Traffic \& Transportation, Vol. 21, No. 5, Sept. 2009, pp. 319-329.

${ }^{5}$ Clarke, J.-P. B., Ho, N. T., Ren, L., Brown, J. A., Elmer, K. R., Tong, K.-O., and Wat, J. K., "Continuous Descent Approach: Design and Flight Test for Louisville International Airport," Journal of Aircraft, Vol. 41, No. 5, 2004, pp. $1054-1066$.

6 "FAA Aerospace Forecast Fiscal Years 2012-2032," Federal Aviation Administration, 2011.

${ }^{7}$ Lauderdale, T. A., Cone, A. C., and Bowe, A. R., "Relative Significance of Trajectory Prediction Errors on an Automated Separation Assurance Algorithm," Proceedings of the 9th USA/Europe Air Traffic Management RESD Seminar, June 2011.

${ }^{8} \mathrm{Wu}$, M. G. and Green, S. M., "Analysis of Fixed Flight Path Angle Descents for the Efficient Descent Advisor," NASA/TM-2011-215992, Nov. 2011.

${ }^{9}$ Tong, K.-O., Schoemig, E., Boyle, D., Scharl, J., and Haraldsdottir, A., "Descent Profile Options for Continuous Descent Arrival Procedures within 3d Path Concept," Proceedings of the IEEE/AIAA 26th Digital Avionics Systems Conference, Oct. 2007, pp. 3.A.3-1 - 3.A.3-11.

${ }^{10}$ Izumi, K. H., "Sensitivity Studies of 4D Descent Strategies in an Advanced Metering Environment," Proceedings of the American Control Conference, 1986, June 1986, pp. 687-692.

${ }^{11}$ Izumi, K. H., Schwab, R. W., Groce, J. L., and Coote, A., "An Evaluation of Descent Strategies for TNAV-Equipped Aircraft in an Advanced Metering Environment ATOPS," Tech. Rep. NASA CR-178093, Boeing Commercial Airplane Company, Dec. 1986.

${ }^{12}$ Chakravarty, A., "Four-Dimensional Fuel-Optimal Guidance in the Presence of Winds," Proceedings of the AIAA Guidance and Control Conference, AIAA-1983-2242, Gatlinburg, Tenn., 1983.

${ }^{13}$ Landry, S., Farley, T., Foster, J., Green, S., Hoang, T., and Wong, G. L., "Distributed Scheduling Architecture for Multi-Center Time-Based Metering," Proceedings of the AIAA Aviation Technology, Integration, and Opterations Conference, AIAA-2003-6758, Nov. 2003.

${ }^{14}$ Swenson, H. N., Hoang, T., Engelland, S., Vincent, D., Sanders, T., Sanford, B., and Heere, K., "Design and Operational Evaluation of the Traffic Management Advisor at the Fort Worth Air Route Traffic Control Center," Proceedings of the 1st USA/Europe Air Traffic Management RED Seminar, June 1997.

${ }^{15}$ Coppenbarger, R. A., Nagle, G., Sweet, D., and Hayashi, M., "The Efficient Descent Advisor: Technology Validation and Transition," Proceedings of the AIAA Aircraft Technology, Integration, and Opterations Conference, Sept. 2012 (to be published).

${ }^{16}$ Nagle, G., Sweet, D., Carr, G., Felipe, V., Trapani, A., Coppenbarger, R., and Hayashi, M., "Human-in-the-Loop Simulation of the Efficient Descent Advisor for 3D Path Arrival Management," Proceedings of the AIAA Aircraft Technology, Integration, and Opterations Conference, AIAA-2011-6877, Virginia Beach, VA, 2011.

${ }^{17}$ Coppenbarger, R., Dyer, G., Hayashi, M., Lanier, R., Stell, L., and Sweet, D., "Development and Testing of Automation for Efficient Arrivals in Constrained Airspace," Proceedings of the 27th International Congress of the Aeronautical Sciences, ICAS2010-11.11.3, Nice, France, 2010.

${ }^{18}$ Coppenbarger, R. A., Lanier, R., Sweet, D., and Dorsky, S., "Design and Development of the En Route Descent Advisor (EDA) for Conflict-Free Arrival Metering," Proceedings of the AIAA Guidance, Navigation, and Control Conference, AIAA2004-4875, Aug. 2004.

${ }^{19}$ Mueller, K. T., Schleicher, D. R., and Coppenbarger, R. A., "Improved Aircraft Path Stretch Algorithms for the En Route Descent Advisor (EDA)," Proceedings of the AIAA Guidance, Navigation, and Control Conference, AIAA-2003-5571, Aug. 2003.

${ }^{20}$ Green, S. M. and Vivona, R. A., "En Route Descent Advisor Concept for Arrival Metering," Proceedings of the AIAA Guidance, Navigation, and Control Conference, AIAA-2001-4114, Aug. 2001. 
${ }^{21}$ Benjamin, S. G., Brown, J. M., Brundage, K. J., Schwartz, B., Smirnova, T., Smith, T. L., Morone, L. L., and Dimego, G., "The Operational RUC-2. Preprints," Proceedings of the 16th Conference on Weather Analysis and Forecasting, Amer. Meteor. Soc., 1998, pp. 249-252.

${ }^{22}$ Lee, A. G., Bouyssounouse, X., and Murphy, J. R., "The Trajectory Synthesizer Generalized Profile Interface," Proceedings of the 10th AIAA Aviation Technology, Integration, and Operations Conference, AIAA-2010-9138, Sept. 2010.

${ }^{23}$ Slattery, R. and Zhao, Y., "Trajectory Synthesis for Air Traffic Automation," Journal of Guidance, Control and Dynamics, Vol. 20, No. 2, March 1997, pp. 232-238.

${ }^{24}$ Erzberger, H., Davis, T. J., and Green, S. M., "Design of Center-TRACON Automation System," Machine Intelligence in Air Traffic Management, Andre Benoit, ed., AGARD CP-538, Oct. 1993, pp. 11-1-11-12.

${ }^{25}$ Nuic, A., Poinsot, C., Iagaru, M.-G., Gallo, E., Navarro, F. A., and Querejeta, C., "Advanced Aircraft Performance Modeling for ATM: Enhancements to the BADA Model," Proceedings of the IEEE/AIAA 24th Digital Avionics Systems Conference, Nov. 2005.

${ }^{26}$ Nikoleris, A. N., Chatterji, G. B., and Coppenbarger, R. A., "Comparison of Fuel Consumption of Alternative Descent Trajectories under Arrival Metering," Proceedings of the AIAA Guidance, Navigation, and Control Conference, AIAA-20124818, Aug. 2012. 